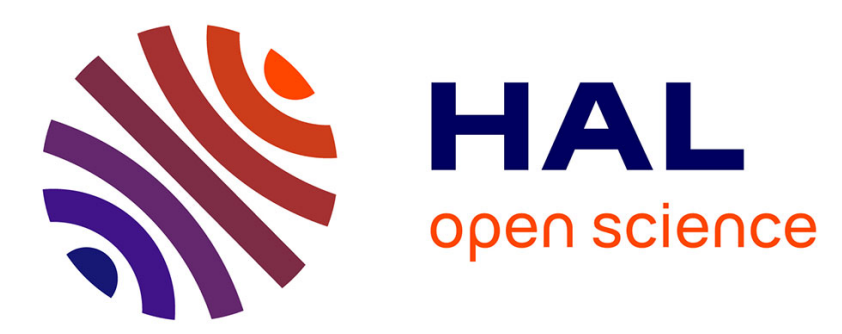

\title{
Influence of the spatial correlation structure of an elastic random medium on its scattering properties
}

\author{
Shahram Khazaie, Régis Cottereau, Didier Clouteau
}

\section{To cite this version:}

Shahram Khazaie, Régis Cottereau, Didier Clouteau. Influence of the spatial correlation structure of an elastic random medium on its scattering properties. Journal of Sound and Vibration, 2016, 370, pp.132-148. 10.1016/j.jsv.2016.01.012 . hal-01281405

\section{HAL Id: hal-01281405 \\ https://hal-centralesupelec.archives-ouvertes.fr/hal-01281405}

Submitted on 2 Mar 2016

HAL is a multi-disciplinary open access archive for the deposit and dissemination of scientific research documents, whether they are published or not. The documents may come from teaching and research institutions in France or abroad, or from public or private research centers.
L'archive ouverte pluridisciplinaire HAL, est destinée au dépôt et à la diffusion de documents scientifiques de niveau recherche, publiés ou non, émanant des établissements d'enseignement et de recherche français ou étrangers, des laboratoires publics ou privés. 


\title{
Influence of the spatial correlation structure of an elastic random medium on its scattering properties ${ }^{\frac{1}{4}}$
}

\author{
S. Khazaie ${ }^{\mathrm{a}, \mathrm{b}}$, R. Cottereau ${ }^{\mathrm{a}, *}$, D. Clouteau ${ }^{\mathrm{a}}$ \\ ${ }^{a}$ MSSMat, CNRS, CentraleSupélec, Université Paris-Saclay, 92290 Châtenay-Malabry, France \\ ${ }^{b}$ Laboratoire M2P2 UMR 7340, Aix-Marseille Université, CNRS, École Centrale Marseille, France
}

\begin{abstract}
In the weakly heterogeneous regime of elastic wave propagation through a random medium, transport and diffusion models for the energy densities can be set up. In the isotropic case, the scattering cross sections are explicitly known as a function of the wave number and the correlations of the Lamé parameters and density. In this paper, we discuss the precise influence of the correlation structure on the scattering cross sections, mean free paths and diffusion parameter, and separate that influence from that of the correlation length and variance. We also analyze the convergence rates towards the low- and high-frequency ranges. For all analyses, we consider five different correlation structures, that allow us to explore a wide range of behaviors. We identify that the controlling factors for the low-frequency behavior are the value of the Power Spectral Density Function (PSDF) and its first non-vanishing derivative at the origin. In the high frequency range, the controlling factor is the third moment of the PSDF (which may be unbounded).
\end{abstract}

Keywords: Elastic wave propagation, Radiative transfer equation, Waves in random media, Correlation, Scattering cross-section

\section{Introduction}

Exploration of the structure of the Earth using seismograms recorded at the surface is a classical problem in geophysics. Its applications range from academic understanding of the interior of the Earth to more industrially-oriented questions related to oil exploration or $\mathrm{CO}_{2}$ and nuclear waste sequestration. The seismograms typically start with a ballistic or coherent part corresponding to the first arrivals of $\mathrm{P}, \mathrm{S}$ or Rayleigh waves. This coherent signal is then followed by an incoherent wave train called the Coda [1,2], with less definite and more randomized features. The analysis of short-period seismograms shows the predominance of multiply scattered waves in this part of the seismograms [3]. At long lapse times, a diffusion regime steps in, which is characterized by the equipartition of energies [4]. The time decay of the late arriving waves is then shown to be a characteristic of the medium, independent of the source and path effects as well as site conditions [5], which makes the Coda a good candidate for identification procedures. Since the original studies on the origin of the Coda [1,5], the spatial variation of the medium properties has been taken into consideration and stochastic modeling has been used as a tool for understanding the complexity of the wave fields in geophysical applications. Recently, there has been an outburst of papers numerically simulating wave propagation in random geophysical media $[6,7,8,9,10]$ to complement the more classical theoretical considerations [11, 12, 13, 14, 3] for the study of the Coda. Similar trends are observed in other fields of physics and engineering, where the decaying part of the signal is analyzed in terms of diffusion equation and used to gather information on the material. This is particularly true for analyses of the propagation of ultrasound in polycrystalline materials $[15,16]$, room acoustics $[17,18,19]$ and high-frequency vibrations of complex structures [20]. Other examples include non-destructive testing of concrete samples [21] and wave propagation in granular materials [22, 23, 24].

\footnotetext{
ฟhis work was partially done within the SINAPS@ project, which benefited from French state funding managed by the National Research Agency under program RNSR Future Investments bearing reference No. ANR-11-RSNR- 0022-04.

*regis.cottereau@centralesupelec.fr
} 
To model wave propagation in random media, three typical length scales are of interest: (i) the dominant wavelength $\lambda$; (ii) the typical size of the heterogeneities (correlation length $\ell_{c}$ for instance); and (iii) the propagation distance $L$. The parameters that are used to separate between the different scattering regimes are $\ell_{c} / \lambda$ and $L / \lambda$ [14]. At least three important regimes can be identified: (1) The effective medium or homogenization regime in which $\ell_{c} / \lambda \ll 1$ and $L / \lambda \sim 1$; (2) the weakly heterogeneous or stochastic scattering regime in which $\ell_{c} / \lambda \sim 1$ and $L / \lambda \gg 1$ (with limited strength of the heterogeneities); and (3) the strongly heterogeneous white-noise regime in which $\ell_{c} / \lambda \ll 1$ and $L / \lambda \gg 1$. In the homogenization regime, a deterministic effective wave equation describes the wave propagation, where the effective stiffness is obtained as in classical stochastic homogenization in statics and the effective density is obtained by arithmetic averaging $[25,26]$. In the strongly heterogeneous regime, the impact of the strong heterogeneities is limited by the disparity in typical lengths. However, an asymptotic regime arises over long enough distances [14] which is mainly influenced by the variance and correlation lengths of the mechanical parameters of the wave equation. Finally, in the weakly heterogeneous regime, the similarity between the wavelength and the heterogeneity length ensures an efficient interaction between the wave and the medium but the strength of the fluctuations is limited so the waves do not become localized [27, 13, 28]. We concentrate in this paper on this weakly heterogeneous regime, which is influenced by the full power spectrum of the mechanical parameters, rather than only on the correlation length and variance.

The theory of radiative transfer of elastic waves is an approach to study the multiple scattering of waves in this regime $[11,15,29,30,16,31]$. The radiative transfer equations (RTE) describe the spatio-temporal evolution of the wave vector-dependent energy densities of the waves. Vector transport equations were firstly developed for polarized light waves in statistically isotropic media by Chandrasekhar [11]. They were later developed for elastic waves in isotropic media independently by Weaver [15] and Ryzhik et al. [31] with different approaches. In these equations, the scattering effects resulting from the randomness of the medium are described by scattering cross-sections, that are explicitly related to the power spectrum of the random mechanical parameters of the wave equation (density and Lamé parameters). Such power spectra are parameterized in general by a variance and a correlation length. However, these two numbers are not sufficient to describe completely a correlation model.

The objective of this paper is to discuss the influence of the correlation structure (that is to say the correlation normalized by the correlation length and variance) on the scattering cross sections. In particular, we want to quantify how this influence compares to that of the variance and correlation length. This work was partially done by various authors in the scalar case [13,3] but we wish to address the elastic case, for which scattering cross sections are much more complicated (see Section 2.5). In particular the existence of $\mathrm{P}$ and $\mathrm{S}$ modes for wave propagation in the background medium means that several cross-sections must be defined rather than just one. Understanding of the precise influence of the correlation structure is important for at least two kinds of applications: (i) for parameterization of the random medium in the context of an inverse problem, and (ii) for design and creation of new meta-materials. When performing an inverse problem in a random medium, it is a priori necessary to identify the complete correlation structure. However, if the analysis shows that in the particular range of frequencies considered only a finite number of parameters of that correlation structure are significant, the complexity of the inverse problem can be drastically reduced. In the case of design of meta-materials, which comes down to an optimization problem, it is again crucial to have a good parameterization of the material to limit the complexity of the problem. Previous authors have tried to tackle this problem by considering simplifying assumptions [32] or one specific correlation model [33, 3], but we concentrate here on comparison between several very classical correlation structures.

The outline of the paper is the following. In Section 2, we introduce the general wave equation and RTE, along with the scattering cross-sections. We also introduce five classical correlation structures (exponential, power-law, Gaussian, triangular and low-pass white noise) that will be compared throughout the paper. We will see that this set of correlation structures shows a wide range of behavior for the scattering cross sections. In the following section (Section 3), we study the influence of the power spectrum structure on the scattering cross-sections and perform the asymptotic analyses. The following section (Section 4) introduces the diffusion approximation and proposes similar analyses for the diffusion parameters.

\section{Elastic wave propagation in isotropic random media}

We consider the propagation of elastic waves in a medium with continuous isotropic random heterogeneities and locally isotropic constitutive behavior [34, 12, 13, 14, 3]. The consideration of discrete random media is possible by 
considering the appropriate correlation structures, but will not be discussed here (see for instance [35, 36, 37, 38]). Likewise, the anisotropic case is fully treated in Baydoun et al. [39] but will be left aside here.

\subsection{Equation of motion and mechanical parameters}

The elastic wave equation in a non-dissipative medium can be written in terms of the displacement field $\mathbf{u}(\mathbf{x}, t)$ as:

$$
\rho \frac{\partial^{2}}{\partial t^{2}} \mathbf{u}(\mathbf{x}, t)-\nabla_{\mathbf{x}} \cdot\left(C(\mathbf{x}): \nabla_{\mathbf{x}} \otimes \mathbf{u}(\mathbf{x}, t)\right)=S(\mathbf{x}, t)
$$

where $\rho$ is the density (assumed constant), $C(\mathbf{x})=C_{i j k l}(\mathbf{x})$ is the fourth-order elastic tensor, that depends continuously on the position $\mathbf{x}$, and $S(\mathbf{x}, t)$ is a given bulk loading field. For simplicity, we neglect here the influence of density variations. The material is assumed isotropic, so that $C_{i j k l}(\mathbf{x})=\lambda(\mathbf{x}) \delta_{i j} \delta_{k l}+\mu(\mathbf{x})\left(\delta_{i k} \delta_{j l}+\delta_{i l} \delta_{j k}\right)$, where $\lambda(\mathbf{x})$ and $\mu(\mathbf{x})$ are the Lamé parameter fields and $\delta_{i j}$ is Kronecker's delta $\left(\delta_{i j}=1\right.$ if $i=j$, and $\delta_{i j}=0$ if $i \neq j$ ). The local velocities of $\mathrm{P}$ and $\mathrm{S}$ waves are related to the local values of Lamé parameters as $v_{p}(\mathbf{x})=\sqrt{(\lambda(\mathbf{x})+2 \mu(\mathbf{x})) / \rho}$ and $v_{s}(\mathbf{x})=\sqrt{\mu(\mathbf{x}) / \rho}$. For each of these two modes, the velocities relate the local frequency and the local wave numbers through the dispersion relations: $\omega=v_{p}\left|\mathbf{k}_{p}\right|=v_{s}\left|\mathbf{k}_{s}\right|$, where the local frequency and wave numbers are respectively the Fourier relatives of time and space. We also introduce the ratio between the space-averaged values of the phase velocities defined as $K=v_{p} / v_{s}$, that is a critical parameter in our study.

In seismology, that is of particular interest for the authors, the values of $K$ for the upper mantle are measured between 1.65 and 1.8 [41]. In laboratory tests under pertinent pressure and temperature conditions for the geological layers, its values can be found between 1.7 and 2.3 [41]. A characteristic value of $K=\sqrt{3}$ is often proposed in the geophysics literature [3] and thermodynamical theory states that its minimum is $\sqrt{4 / 3} \approx 1.16$.

\subsection{Random model of the mechanical parameters}

We consider a random description of the mechanical parameters $\lambda(\mathbf{x})$ and $\mu(\mathbf{x})$. We model them as second-order statistically homogeneous random fields and specify their mean values $\underline{\lambda}$ and $\mu$, autocorrelation functions (ACF) $\mathrm{R}_{\lambda}\left(\mathbf{x}-\mathbf{x}^{\prime}\right)=\mathbb{E}\left[(\lambda(\mathbf{x})-\underline{\lambda})\left(\lambda\left(\mathbf{x}^{\prime}\right)-\underline{\lambda}\right)\right]$ and $\mathrm{R}_{\mu}\left(\mathbf{x}-\mathbf{x}^{\prime}\right)=\mathbb{E}\left[(\mu(\mathbf{x})-\mu)\left(\bar{\mu}\left(\mathbf{x}^{\prime}\right)-\bar{\mu}\right)\right]$, and cross-correlation function $\mathrm{R}_{\lambda \mu}\left(\mathbf{x}-\mathbf{x}^{\prime}\right)=\mathbb{E}\left[(\lambda(\mathbf{x})-\underline{\lambda})\left(\mu\left(\mathbf{x}^{\prime}\right)-\bar{\mu}\right)\right]$. The fact that the averages do not depend on the space position and that the correlation functions only depend on $\left(\mathbf{x}-\mathbf{x}^{\prime}\right)$ is a consequence of the hypothesis of statistical homogeneity. Further, the isotropy hypothesis means that these correlation functions only depend on the distance between the points $r=\left|\mathbf{x}-\mathbf{x}^{\prime}\right|$ so that they can be modeled as 1D functions. Models of a correlation function $\mathrm{R}(r)$ are usually introduced after normalization by the variance $v^{2}=\mathrm{R}(0)$ and the correlation length $\ell_{c}$. The latter is defined as

$$
\ell_{c}=\frac{2}{v^{2}} \int_{\mathbb{R}^{+}} \mathrm{R}(r) d r .
$$

We define the non-dimensional correlation function as $\hat{\mathrm{R}}(\eta)=\mathrm{R}\left(\eta \ell_{c}\right) / v^{2}$. For the simplicity of the presentation, we will consider from now on that all the correlation lengths are the same:

$$
\frac{2}{v_{\lambda}^{2}} \int_{\mathbb{R}^{+}} \mathrm{R}_{\lambda}(r) d r=\frac{2}{v_{\mu}^{2}} \int_{\mathbb{R}^{+}} \mathrm{R}_{\mu}(r) d r=\frac{2}{v_{\lambda \mu}^{2}} \int_{\mathbb{R}^{+}} \mathrm{R}_{\lambda \mu}(r) d r \equiv \ell_{c},
$$

as well as the normalized correlation functions: $\hat{\mathrm{R}}_{\lambda}(\eta)=\hat{\mathrm{R}}_{\mu}(\eta)=\hat{\mathrm{R}}_{\lambda \mu}(\eta) \equiv \hat{\mathrm{R}}(\eta)$. The different correlation models therefore only differ through the variances $v_{\lambda}^{2}=\mathrm{R}_{\lambda}(0)$ and $v_{\mu}^{2}=\mathrm{R}_{\mu}(0)$ and the coefficient $v_{\lambda \mu}=\mathrm{R}_{\lambda \mu}(0)$. Finally, we introduce the normalized power spectral density function (PSDF) $\Phi(\zeta)$ as the Fourier transform of $\hat{R}(\eta)$. We consider the classical definition of the Fourier transform in spherical coordinates for isotropic functions in 3D:

$$
\Phi(\zeta)=\frac{1}{2 \pi^{2}} \int_{\mathbb{R}^{+}} \operatorname{sinc}(\zeta \eta) \eta^{2} \hat{R}(\eta) d \eta .
$$

in which $\operatorname{sinc}(x)=\sin (x) / x$. Note that the value of the PSDF at the origin is related to the second moment of the $\operatorname{ACF} \Phi(0)=\int_{\mathbb{R}^{+}} \eta^{2} \hat{R}(\eta) d \eta / 2 \pi^{2}$ and that the first moment of the PSDF is related to the average of the ACF, which is a constant in the normalized case $\int_{\mathbb{R}^{+}} \zeta \Phi(\zeta) d \zeta=\int_{\mathbb{R}^{+}} \hat{R}(\eta) d \eta / 2 \pi^{2}=1 / 4 \pi^{2}$. These last two identities will be used extensively to investigate the asymptotic behavior of scattering parameters. 

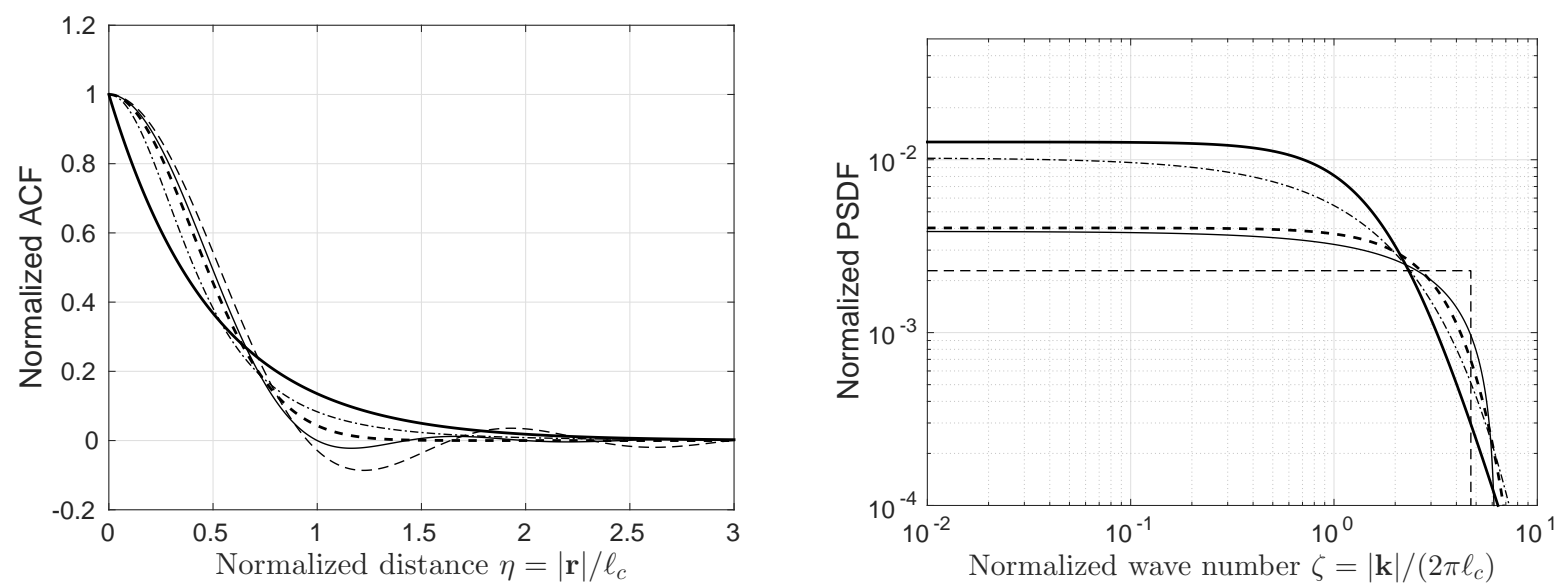

Figure 1: Classical correlation functions (left figure) and corresponding power spectral densities (right figure), as described in Table 1: exponential (thick solid line), power-law (thin dashed-dotted line), Gaussian (thick dashed line), triangular (thin solid line) and low-pass white noise (thin dashed line).

\subsection{Classical correlation models}

We now introduce several models of correlation that have been used in the literature (see for instance in geophysics [42]) and will be discussed in this paper: exponential, power-law, Gaussian, triangular, and low-pass white noise. They are defined in Table 1 in their non-dimensional form, that is to say such that $\hat{\mathrm{R}}(0)=1$ and $2 \int_{\mathbb{R}^{+}} \hat{\mathrm{R}}(\eta) d \eta=$ 1. The ACF and PSDF for these models are drawn in Figure 1. One realization of a centered unit Gaussian random field with each of the correlation models is also plotted in Figure 2 to give an idea of the difference of texture that these correlation models imply.

Table 1: Definitions of the normalized correlation models. The Heaviside function $H(\zeta)$ is such that $\mathrm{H}(\zeta \geq 0)=1$ and $H(\zeta<0)=0$.

\begin{tabular}{|c|c|c|c|c|}
\hline $\begin{array}{c}\text { Correlation } \\
\text { model }\end{array}$ & $\begin{array}{c}\text { Normalized ACF } \\
\hat{R}(\eta)\end{array}$ & $\begin{array}{c}\text { Normalized PSDF } \\
\Phi(\zeta)\end{array}$ & $\begin{array}{c}\text { Low freq. approx. } \\
\Phi(\zeta \ll 1)\end{array}$ & $\begin{array}{c}\text { High freq. approx. } \\
\Phi(\zeta \gg 1)\end{array}$ \\
\hline \hline Exponential & $\exp (-2 \eta)$ & $\frac{1}{8 \pi^{2}\left(1+\frac{\zeta^{2}}{4}\right)^{2}}$ & $\frac{1}{8 \pi^{2}}\left(1-\frac{\zeta^{2}}{2}\right)+\mathrm{o}\left(\zeta^{2}\right)$ & $\frac{2}{\pi^{2}} \zeta^{-4}+\mathrm{o}\left(\zeta^{-4}\right)$ \\
\hline Power-law & $\frac{1}{\left(1+\frac{\pi^{2} \eta^{2}}{4}\right)^{2}}$ & $\frac{1}{\pi^{4}} \exp \left(-2 \frac{\zeta}{\pi}\right)$ & $\frac{1}{\pi^{4}}\left(1-\frac{2 \zeta}{\pi}\right)+\mathrm{o}(\zeta)$ & spectral \\
\hline Gaussian & $\exp \left(-\pi \eta^{2}\right)$ & $\frac{1}{8 \pi^{3}} \exp \left(-\frac{\zeta^{2}}{4 \pi}\right)$ & $\frac{1}{8 \pi^{3}}\left(1-\frac{\zeta^{2}}{4 \pi}\right)+\mathrm{o}\left(\zeta^{2}\right)$ & spectral \\
\hline Triangular & $\frac{12(2-2 \cos (2 \pi \eta)-(2 \pi \eta) \sin (2 \pi \eta))}{(2 \pi \eta)^{4}}$ & $\frac{3}{8 \pi^{4}}\left(1-\frac{\zeta}{2 \pi}\right) \mathrm{H}(2 \pi-\zeta)$ & $\frac{3}{8 \pi^{4}}\left(1-\frac{\zeta}{2 \pi}\right)$ & 0 \\
\hline $\begin{array}{c}\text { Low-pass } \\
\text { white noise }\end{array}$ & $\frac{3\left(\sin \left(\frac{3 \pi}{2} \eta\right)-\frac{3 \pi}{2} \eta \cos \left(\frac{3 \pi}{2} \eta\right)\right)}{\left(\frac{3 \pi}{2} \eta\right)^{3}}$ & $\frac{2}{9 \pi^{4}} \mathrm{H}\left(\frac{3 \pi}{2}-\zeta\right)$ & $\frac{2}{9 \pi^{4}}$ & 0 \\
\hline
\end{tabular}

Other correlation models could have been chosen for this study. However, we believe these five present a wide range of behaviors, representative of a larger set of models. In particular, the influence of the boundedness of the support of $\Phi(\zeta)$ will be significant (it is bounded for triangular and low-pass white noise models, and unbounded for the others), as well the values of its derivative at the origin $\left(\Phi^{\prime}(0)=0\right.$ for the exponential and Gaussian models, and $\Phi^{(n)}(0)=0$, for all $n \geq 1$ for the low-pass white noise model).

\subsection{Multiple scattering and diffusion of elastic waves}

The RTE of elastic waves describe the propagation of the energy densities of the $\mathrm{P}$ and $\mathrm{S}$ waves. They are derived using a multiscale expansion of the Wigner transform of the wave field in the stochastic regime $\left(\ell_{c} \sim \lambda, L \gg \lambda\right.$ and 


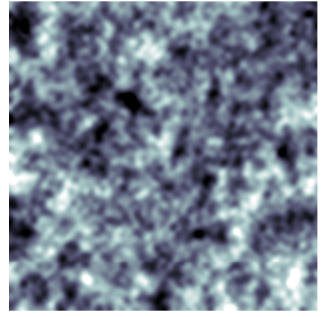

(a) Exponential

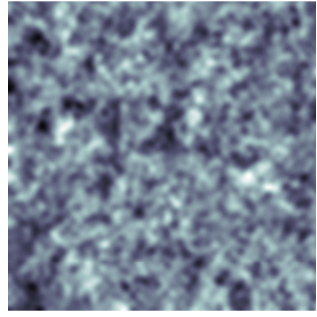

(b) Power law

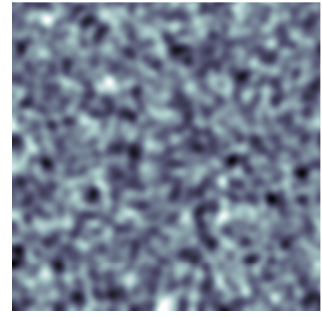

(c) Gaussian

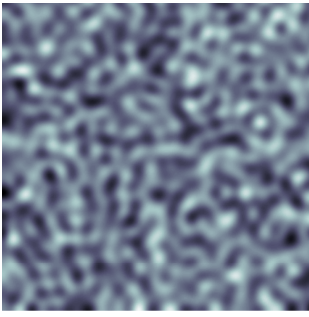

(d) Triangular

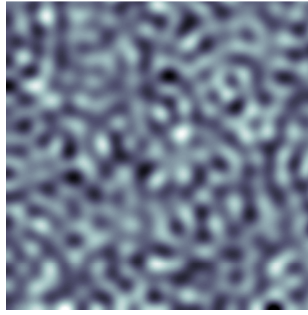

(e) Low-pass white noise

Figure 2: One realization of a centered unit Gaussian random field with different correlation models: (a) exponential, (b) power-law, (c) Gaussian, (d) triangular, and (e) low-pass white noise. The images are $L \times L$, where $L=20 \ell_{c}$ and $\ell_{c}$ is the correlation length.

$\left.v^{2} \ll 1\right)$. For random media with local isotropy [15, 31], they are set as the following coupled system of equations:

$$
\frac{\partial a^{p}(\mathbf{k})}{\partial t}-\left\{\omega^{p}, a^{p}(\mathbf{k})\right\}=\int_{\mathbb{R}^{3}} \sigma_{\mathrm{PP}}\left(\mathbf{k}, \mathbf{k}^{\prime}\right) a^{p}\left(\mathbf{k}^{\prime}\right) d \mathbf{k}^{\prime}-\Sigma_{\mathrm{PP}}(\mathbf{k}) a^{p}(\mathbf{k})+\int_{\mathbb{R}^{3}} \sigma_{\mathrm{PS}}\left(\mathbf{k}, \mathbf{k}^{\prime}\right)\left[a^{s}\left(\mathbf{k}^{\prime}\right)\right] d \mathbf{k}^{\prime}-\Sigma_{\mathrm{SP}}(\mathbf{k}) a^{p}(\mathbf{k})
$$

$$
\begin{aligned}
\frac{\partial\left[a^{s}(\mathbf{k})\right]}{\partial t}-\left\{\omega^{s},\left[a^{s}(\mathbf{k})\right]\right\}+ & {\left[a^{s}(\mathbf{k})\right] N-N\left[a^{s}(\mathbf{k})\right] } \\
= & \int_{\mathbb{R}^{3}} \sigma_{\mathrm{SS}}\left(\mathbf{k}, \mathbf{k}^{\prime}\right)\left[a^{s}\left(\mathbf{k}^{\prime}\right)\right] d \mathbf{k}^{\prime}-\Sigma_{\mathrm{SS}}(\mathbf{k})\left[a^{s}(\mathbf{k})\right]+\int_{\mathbb{R}^{3}} \sigma_{\mathrm{SP}}\left(\mathbf{k}, \mathbf{k}^{\prime}\right) a^{p}\left(\mathbf{k}^{\prime}\right) d \mathbf{k}^{\prime}-\Sigma_{\mathrm{PS}}(\mathbf{k})\left[a^{s}(\mathbf{k})\right]
\end{aligned}
$$

where $a^{p}(t, \mathbf{x}, \mathbf{k})$ and $\left[a^{s}(t, \mathbf{x}, \mathbf{k})\right]$ are respectively the scalar directional energy density of compressional $\mathrm{P}$ waves and the $2 \times 2$ coherence matrix containing the directional energy densities of the coupled shear $\mathrm{S}$ waves. The Poisson bracket is defined as $\{f, g\}=\nabla_{\mathbf{x}} f \cdot \nabla_{\mathbf{k}} g-\nabla_{\mathbf{k}} f \cdot \nabla_{\mathbf{x}} g$. When the background medium is homogeneous, the velocity does not depend on the space variable and the Poisson brackets reduce to the classical convection terms $v_{p} \hat{\mathbf{k}} \cdot \nabla_{\mathbf{x}} a^{p}(t, \mathbf{x}, \mathbf{k})$ and $v_{s} \hat{\mathbf{k}} \cdot \nabla_{\mathbf{x}}\left[a^{s}(t, \mathbf{x}, \mathbf{k})\right]$. The $2 \times 2$ matrix $N(\mathbf{x}, \mathbf{k})$ describes the polarization effect of shear waves [31] and vanishes when the background medium is homogeneous. The differential and total scattering cross sections $\sigma_{\mathrm{IJ}}\left(\mathbf{k}, \mathbf{k}^{\prime}\right)$ and $\Sigma_{\mathrm{IJ}}(\mathbf{k})$, for I, J $\in\{\mathrm{P}, \mathrm{S}\}$, describe the complex interactions of the wave energy with the heterogeneities of the medium and its subsequent change of path and/or mode transformation. These operators are described in more details below.

\subsection{Definition and normalizations of the scattering cross-sections}

The differential scattering cross sections $\sigma_{\mathrm{IJ}}\left(\mathbf{k}, \mathbf{k}^{\prime}\right.$ ) (with unit $\mathrm{m}^{3} / \mathrm{s}$ ) represent the rate at which incident energy of mode type $\mathbf{J}$ with wave vector $\mathbf{k}^{\prime}$ is converted to scattered wave energy of mode type I with wave vector $\mathbf{k}$. The total scattering cross sections $\Sigma_{\mathrm{IJ}}(\mathbf{k})$ (with unit $1 / \mathrm{s}$ )

$$
\Sigma_{\mathrm{IJ}}(\mathbf{k})=\int_{\mathbb{R}^{3}} \sigma_{\mathrm{IJ}}\left(\mathbf{k}, \mathbf{k}^{\prime}\right) d \mathbf{k}^{\prime}, \mathrm{I}, \mathrm{J} \in\{\mathrm{P}, \mathrm{S}\}
$$

represent the rate at which incident energy of mode type $\mathbf{J}$ with wave vector $\mathbf{k}$ is converted to scattered wave energy of mode type I in any direction. Finally, the forward scattering cross-sections (with unit 1/s)

$$
\Sigma_{\mathrm{IJ}}^{\prime}(\mathbf{k})=\int_{\mathbb{R}^{3}}\left(\hat{\mathbf{k}} \cdot \hat{\mathbf{k}}^{\prime}\right) \sigma_{\mathrm{IJ}}\left(\mathbf{k}, \mathbf{k}^{\prime}\right) d \mathbf{k}^{\prime}, \mathrm{I}, \mathrm{J} \in\{\mathrm{P}, \mathrm{S}\},
$$

characterize the degree of scattering in the forward direction. Its value can be negative, zero or positive respectively for backward, non-preferential (but not necessarily isotropic) and forward scattering.

We now recall the formulas developed in [31] in a spherical basis, thanks to the assumed isotropy of the medium. They were derived assuming that the incident waves propagate along the vertical direction, with no restriction on the model. The base for the incident wave is then $\left[\hat{\zeta}, z^{(1)}(\hat{\zeta}), z^{(2)}(\hat{\zeta})\right]$, where

$$
\hat{\boldsymbol{\zeta}}=\left(\begin{array}{l}
0 \\
0 \\
1
\end{array}\right) \quad ; \quad z^{(1)}(\hat{\zeta})=\left(\begin{array}{l}
1 \\
0 \\
0
\end{array}\right) \quad ; \quad z^{(2)}(\hat{\zeta})=\left(\begin{array}{l}
0 \\
1 \\
0
\end{array}\right)
$$


and the basis for the scattered wave is taken as $\left[\hat{\zeta}^{\prime}, z^{(1)}\left(\hat{\zeta}^{\prime}\right), z^{(2)}\left(\hat{\zeta}^{\prime}\right)\right]$, where

$$
\hat{\zeta}^{\prime}=\left(\begin{array}{c}
\sin \theta \cos \phi \\
\sin \theta \sin \phi \\
\cos \theta
\end{array}\right) ; \quad z^{(1)}\left(\hat{\zeta}^{\prime}\right)=\left(\begin{array}{c}
\cos \theta \cos \phi \\
\cos \theta \sin \phi \\
-\sin \theta
\end{array}\right) ; \quad z^{(2)}\left(\hat{\zeta}^{\prime}\right)=\left(\begin{array}{c}
-\sin \phi \\
\cos \phi \\
0
\end{array}\right)
$$

Note that we have $\left|\zeta-\zeta^{\prime}\right|=2 \zeta \sin (\theta / 2)$. Using these bases, the differential scattering cross-sections can be normalized in the following way:

$$
\begin{gathered}
\sigma_{\mathrm{PP}}\left(\mathbf{k}, \mathbf{k}^{\prime}\right)\left[a^{p}\left(\mathbf{k}^{\prime}\right)\right]=v_{p} \ell_{c}^{2} \bar{\sigma}_{p p}\left(\frac{|\mathbf{k}| \ell_{c}}{2 \pi}, \theta\right) a^{p}\left(\mathbf{k}^{\prime}\right) \delta\left(\frac{|\mathbf{k}| \ell_{c}}{2 \pi}-\frac{\left|\mathbf{k}^{\prime}\right| \ell_{c}}{2 \pi}\right), \\
\sigma_{\mathrm{SP}}\left(\mathbf{k}, \mathbf{k}^{\prime}\right)\left[a^{p}\left(\mathbf{k}^{\prime}\right)\right]=v_{p} \ell_{c}^{2} \bar{\sigma}_{p s}\left(\frac{|\mathbf{k}| \ell_{c}}{2 \pi K}, \theta\right) G(\phi) a^{p}\left(\mathbf{k}^{\prime}\right) \delta\left(\frac{|\mathbf{k}| \ell_{c}}{2 \pi K}-\frac{\left|\mathbf{k}^{\prime}\right| \ell_{c}}{2 \pi}\right), \\
\sigma_{\mathrm{PS}}\left(\mathbf{k}, \mathbf{k}^{\prime}\right)\left[a^{s}\left(\mathbf{k}^{\prime}\right)\right]=v_{s} \ell_{c}^{2} \bar{\sigma}_{p s}\left(\frac{|\mathbf{k}| \ell_{c}}{2 \pi}, \theta\right) \operatorname{Tr}\left(G(0) a^{s}\left(\mathbf{k}^{\prime}\right)\right) \delta\left(\frac{K|\mathbf{k}| \ell_{c}}{2 \pi}-\frac{\left|\mathbf{k}^{\prime}\right| \ell_{c}}{2 \pi}\right), \\
\sigma_{\mathrm{SS}}\left(\mathbf{k}, \mathbf{k}^{\prime}\right)\left[a^{s}\left(\mathbf{k}^{\prime}\right)\right]=v_{s} \ell_{c}^{2} \bar{\sigma}_{s s}\left(\frac{|\mathbf{k}| \ell_{c}}{2 \pi}, \theta\right) R(\phi) \Gamma(\theta)\left[a^{s}\left(\mathbf{k}^{\prime}\right)\right](R(\phi) \Gamma(\theta))^{T} \delta\left(\frac{|\mathbf{k}| \ell_{c}}{2 \pi}-\frac{\left|\mathbf{k}^{\prime}\right| \ell_{c}}{2 \pi}\right),
\end{gathered}
$$

where

$$
G(\phi)=\left[\begin{array}{cc}
\cos ^{2} \phi & \cos \phi \sin \phi \\
\cos \phi \sin \phi & \sin ^{2} \phi
\end{array}\right], \quad \Gamma(\theta)=\left[\begin{array}{cc}
\cos 2 \theta & 0 \\
0 & \cos \theta
\end{array}\right], \quad R(\phi)=\left[\begin{array}{cc}
\cos \phi & -\sin \phi \\
\sin \phi & \cos \phi
\end{array}\right] .
$$

The Dirac delta functions $\delta(\cdot)$ in the right hand sides of Eq. (11-14) are a consequence of the conservation of frequency during scattering. The normalized functions $\bar{\sigma}_{i j}$ (that will be described in more details below) include influences from the correlation kernel, the variances and the velocity ratio $K$ but not from the correlation length.

The total scattering cross-sections are normalized in the following way:

$$
\begin{gathered}
\Sigma_{\mathrm{PP}}(\mathbf{k})=\frac{v_{p}}{\ell_{c}} \bar{\Sigma}_{p p}\left(\frac{|\mathbf{k}| \ell_{c}}{\pi}\right) \\
\Sigma_{\mathrm{PS}}(\mathbf{k}) \mathbf{I}_{2}=\frac{v_{p}}{\ell_{c}} \bar{\Sigma}_{p s}\left(\frac{|\mathbf{k}| \ell_{c}}{\pi} \sqrt{K_{0} K}\right) \mathbf{I}_{2}=2 K^{3} \Sigma_{\mathrm{SP}}(\mathbf{k} / K) \\
\Sigma_{\mathrm{SS}}(\mathbf{k})=\frac{v_{s}}{\ell_{c}} \bar{\Sigma}_{s s}\left(\frac{|\mathbf{k}| \ell_{c}}{\pi}\right) \mathbf{I}_{2}
\end{gathered}
$$

where $\mathbf{I}_{2}$ is a $2 \times 2$ identity matrix and $K_{0}=(K+1 / K) / 2$. Finally, the forward scattering cross-sections are normalized in a similar way. Note that all the normalized scattering cross sections are now functions of a normalized parameter, ratio of the correlation length and wavelength:

$$
\zeta=\frac{\ell_{c}}{\lambda}=\frac{|\mathbf{k}| \ell_{c}}{2 \pi}
$$

\section{Influence of the correlation function on the scattering parameters}

Now that we have recalled all the relevant definitions, we can start to address the main objective of this paper, which is the characterization of the influence of the correlation structure on the scattering parameters. We will consider successively the influence on (i) the differential scattering cross sections, (ii) the total scattering cross sections, and (iii) the forward scattering cross sections. 


\subsection{Influence of the correlation model on the differential scattering cross-sections}

Assuming that the different correlations only differ by their variance, as discussed in Section 2.2, the normalized differential scattering cross sections are [31]:

$$
\left\{\begin{array}{l}
\bar{\sigma}_{p p}(\zeta, \theta)=\frac{\pi^{2}}{K^{4}} \zeta^{2}\left[\left(K^{2}-2\right)^{2} v_{\lambda}^{2}+4\left(K^{2}-2\right) \cos ^{2} \theta v_{\lambda \mu}+4 \cos ^{4} \theta v_{\mu}^{2}\right] \Phi\left(4 \pi \zeta \sin \frac{\theta}{2}\right), \\
\bar{\sigma}_{p s}(\zeta, \theta)=4 \pi^{2} \zeta^{2} \cos ^{2} \theta \sin ^{2} \theta v_{\mu}^{2} \Phi\left(2 \pi \zeta \sqrt{1+K^{2}-2 K \cos \theta}\right), \\
\bar{\sigma}_{s s}(\zeta, \theta)=\pi^{2} \zeta^{2} v_{\mu}^{2} \Phi\left(4 \pi \zeta \sin \frac{\theta}{2}\right),
\end{array}\right.
$$

Figure 3 presents the polar plots of the normalized differential scattering cross sections. These plots are made assuming that an incident wave comes from the left and is reflected by an object in the center of the plot. The polar plots illustrate the influence of the correlation structure on the directionality of the scattering at different frequencies and for the three differential scattering parameters. Note that Figure 3 corresponds to the particular case of $K=\sqrt{3}$ and $v_{\lambda}=v_{\mu}=v_{\lambda \mu}=0.1$, but similar results are obtained with other combinations (except for extreme cases, such as $K=\sqrt{2}$, that are not relevant here). Note also that we plot in Fig. $3 \hat{\sigma}_{i j}(\zeta, \theta)=\bar{\sigma}_{i j}(\zeta, \theta) / \int_{0}^{2 \pi} \bar{\sigma}_{i j}(\zeta, \theta) d \theta$ rather than $\bar{\sigma}$ in order to be able to make comparisons between the shapes of the differential scattering cross sections. Indeed the maximum amplitude varies with frequency over many orders of magnitude, which would not allow us to compare the shapes as we wish to do here. Note also that normalization with the total scattering cross section, as is usually done in acoustics, is not possible here, because the P-S total scattering cross-section sometimes vanishes (see Fig. 4)

We observe that elastic wave scattering is intrinsically anisotropic (dependent on the scattering angle) at all frequencies. This is even true at low frequencies, when there is symmetry between forward and backward scattering. However, the influence of the correlation structure seems to evolve with the frequency range. At low frequency, it has no influence and the polar plots for the five correlation structures exactly overlap. In that frequency range, which corresponds to infinitely small correlation length, the random media is indeed essentially a white noise, whatever the actual correlation model. At higher frequencies, all polar plots have a tendency to move towards the forward direction, which is reasonable since this frequency range corresponds to more homogeneous media, but this evolution is not similar for all models. In particular, for the P-to-P and S-to-S scattering cross sections, the exponential and triangular correlation models seem to induce faster increase of forward scattering with frequency. On the other hand, the low-pass white noise seems to remain non-preferential for higher frequencies. Finally, it can be observed that at high frequencies, the values of $\hat{\sigma}_{p s}$ vanish for the low-pass white noise and triangular models. It can be simply shown that for these models $\hat{\sigma}_{p s}$ cancels for the frequencies larger than $\zeta_{c} /(2 \pi(K-1))$ where $\zeta_{c}$ is the cutoff frequency of the corresponding spectrum. These remarks will be further quantified through the analysis of the total and forward scattering cross sections, in the next sections.

\subsection{Influence of the correlation model on the total scattering cross-sections}

The normalized total scattering cross sections are defined as:

$$
\left\{\begin{array}{l}
\bar{\Sigma}_{p p}(\zeta)=\frac{4 \pi^{6}}{K^{4}}\left[\left(K^{2}-2\right)^{2} v_{\lambda}^{2} I_{0}(\zeta)+4\left(K^{2}-2\right) v_{\lambda \mu} I_{2}(\zeta)+4 v_{\mu}^{2} I_{4}(\zeta)\right] \zeta^{4} \\
\bar{\Sigma}_{p s}(\zeta)=\frac{16 \pi^{6}}{K K_{0}^{2}} v_{\mu}^{2}\left[J_{2}(\zeta)-J_{4}(\zeta)\right] \zeta^{4} \\
\bar{\Sigma}_{s s}(\zeta)=2 \pi^{6} v_{\mu}^{2}\left[I_{0}(\zeta)-3 I_{2}(\zeta)+4 I_{4}(\zeta)\right] \zeta^{4}
\end{array}\right.
$$

in which

$$
I_{n}(\zeta)=\int_{0}^{1}\left(1-2 \chi^{2}\right)^{n} \chi \Phi(2 \pi \zeta \chi) d \chi, \quad J_{n}(\zeta)=K_{0}^{n+1} \int_{K_{1}}^{K_{2}}\left(1-2 \chi^{2}\right)^{n} \chi \Phi(2 \pi \zeta \chi) d \chi,
$$

and where $K_{1}=\sqrt{\left(1-1 / K_{0}\right) / 2}$ and $K_{2}=\sqrt{\left(1+1 / K_{0}\right) / 2}$. The behavior of these functions $I_{n}(\zeta)$ and $J_{n}(\zeta)$ is studied in Appendix A. Based on the asymptotic behavior of the functions $I_{n}(\zeta)$ and $J_{n}(\zeta)$ described in detail in Appendix A, the asymptotic behavior in the low and high frequency ranges is presented in Table 2 . The total scattering cross sections are also plotted as functions of the normalized frequency $\zeta$ in Figure 4.

It is clear from Table 2 and Figure 4 that the influence of the correlation model on the low-frequency behavior for all total scattering cross sections is fully summarized by the scalar number $\Phi(0)$, which characterizes the lowfrequency content of the correlation kernel. Indeed, on Fig. 2, the texture of the images illustrates that the high 


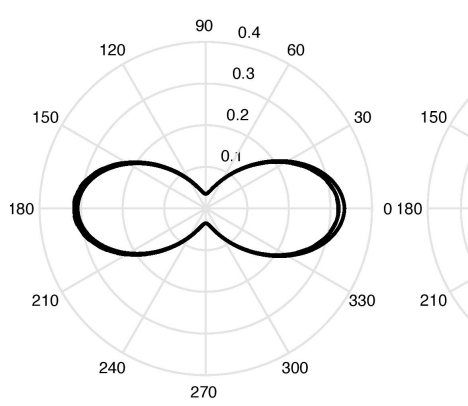

(a) $\hat{\sigma}_{p p}, \zeta=0.01$

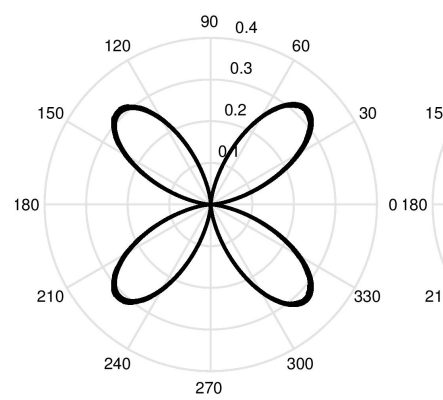

(e) $\hat{\sigma}_{p s}, \zeta=0.01$

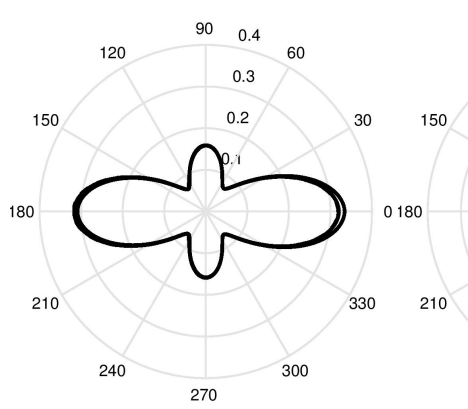

(i) $\hat{\sigma}_{s s}, \zeta=0.01$

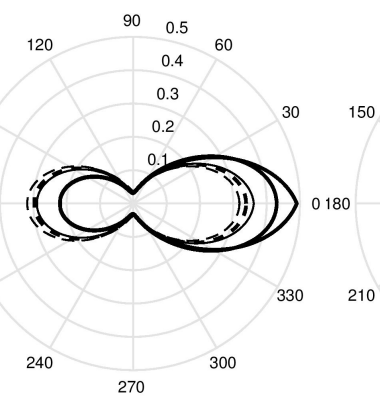

(b) $\hat{\sigma}_{p p}, \zeta=0.1$

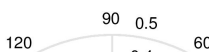

\begin{tabular}{l|l|l}
120 & 60 \\
\hline
\end{tabular}

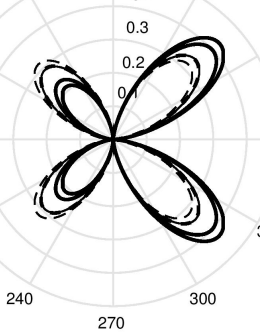

(f) $\hat{\sigma}_{p s}, \zeta=0.1$

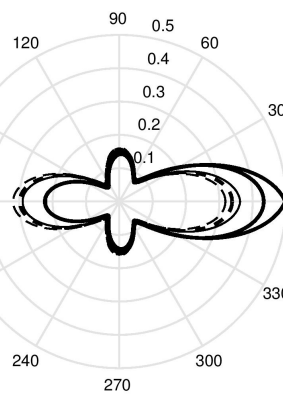

(j) $\hat{\sigma}_{s s}, \zeta=0.1$

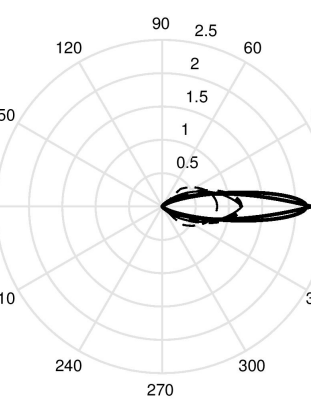

(c) $\hat{\sigma}_{p p}, \zeta=1$

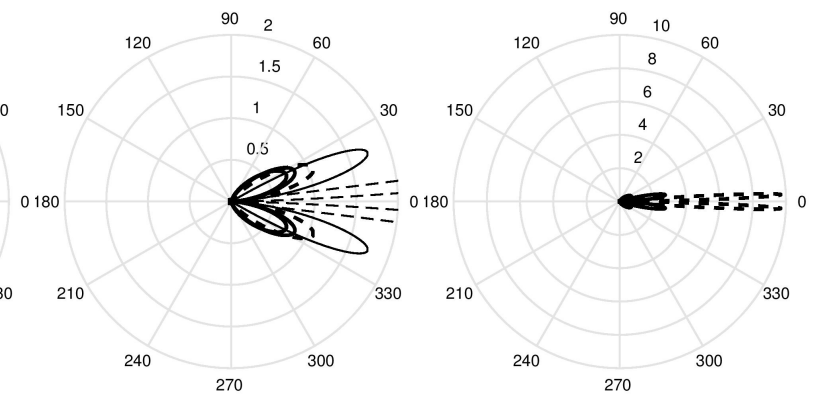

(g) $\hat{\sigma}_{p s}, \zeta=1$

(h) $\hat{\sigma}_{p s}, \zeta=10$

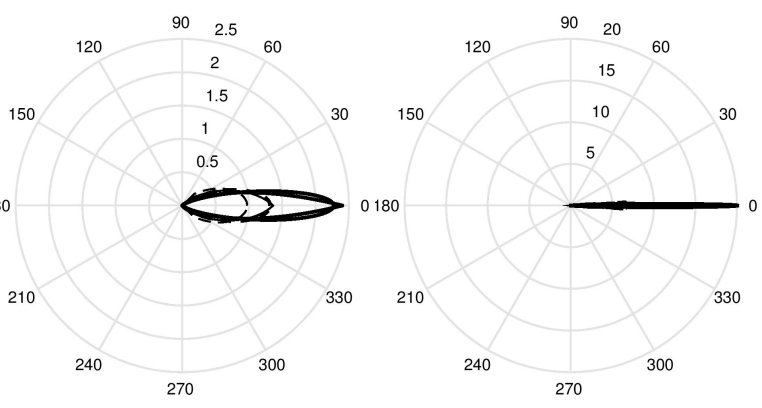

(k) $\hat{\sigma}_{s s}, \zeta=1$

(1) $\hat{\sigma}_{s s}, \zeta=10$

Figure 3: Influence of the correlation model on the scattering probability density functions $\hat{\sigma}_{i j}(\zeta, \theta)=\bar{\sigma}_{i j}(\zeta, \theta) / \int_{0}^{2 \pi} \bar{\sigma}_{i j}(\zeta, \theta) d \theta$ for $K=\sqrt{3}$ and $v_{\lambda}=v_{\mu}=v_{\lambda \mu}=0.1$ : exponential (thick solid line), power-law (thin dashed-dotted line), Gaussian (thick dashed line), triangular (thin solid line) and low-pass white noise (thin dashed line).

frequency content decreases from the exponential model on the left to the low-pass white noise on the right, but the low-frequency content also decreases from the exponential to the low-pass white noise, as illustrated on Fig. 1. In the low-frequency regime, the P-to-P and S-to-S total scattering cross-sections are therefore proportional to the low-frequency content of the correlation kernel. In the high frequency range, there is no influence of the correlation model on the P-to-P and S-to-S total scattering cross sections. However, there is a large influence on the P-to-S total scattering. Indeed, for the models with bounded support (triangular and low-pass white noise), there is a cutoff frequency above which the total scattering cross section vanishes. For the power-law and Gaussian correlation, whose spectrum goes to zero exponentially, the P-to-S total scattering cross-section does likewise. Finally, for the exponential model, there is a plateau in the total scattering cross section and it never vanishes. This is due to the slow decay of the spectrum of the exponential model with frequency. Even when the correlation length is very large compared to the wavelength, there are enough high-frequency fluctuations in the random medium to induce P-to-S scattering. Overall, except this high frequency behavior of the P-to-S cross section, Figure 4 makes it clear that the 


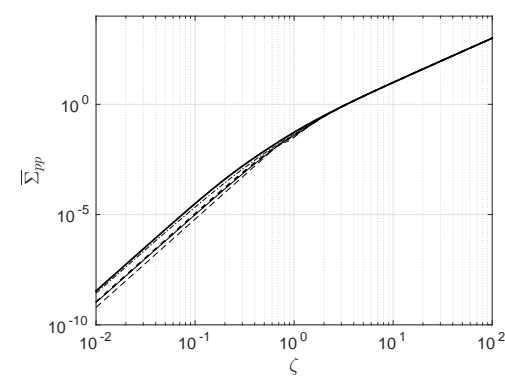

(a) $\bar{\Sigma}_{p p}$

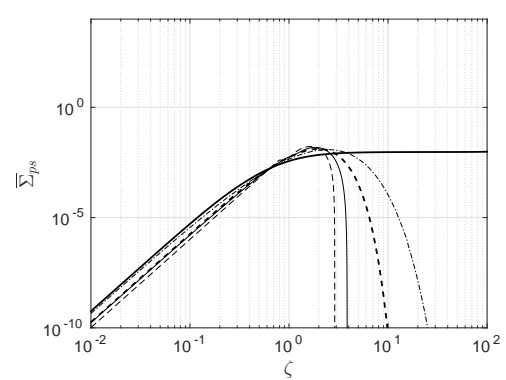

(b) $\bar{\Sigma}_{p s}$

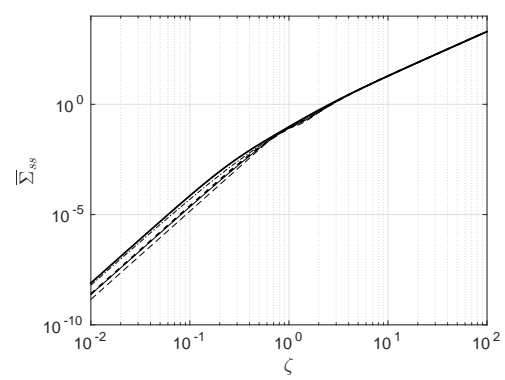

(c) $\bar{\Sigma}_{s s}$

Figure 4: $\bar{\Sigma}_{p p}, \bar{\Sigma}_{p s}$ and $\bar{\Sigma}_{s s}$ in terms of $\zeta$ for $K=\sqrt{3}$ and $v_{\lambda}=v_{\mu}=v_{\lambda \mu}=0.1$ : exponential (thick solid line), power-law (thin dashed-dotted line), Gaussian (thick dashed line), triangular (thin solid line) and low-pass white noise (thin dashed line).

influence of the correlation model is rather limited.

Table 2: Asymptotic behavior of the total and forward scattering cross-sections for different correlation models. The constants $W_{n}^{p}$ and functions $V_{n}^{p}(K)$ are described in Appendix A.1 and Appendix A.2, respectively.

\begin{tabular}{|c|c|c|}
\hline Parameter & Low frequency $\zeta \ll 1$ & High frequency $\zeta \gg 1$ \\
\hline $\bar{\Sigma}_{p p}(\zeta)$ & $\frac{2 \pi^{6}}{K^{4}}\left(\left(K^{2}-2\right)^{2} v_{\lambda}^{2}+\frac{4}{3}\left(K^{2}-2\right) v_{\lambda \mu}+\frac{4}{5} v_{\mu}^{2}\right) \Phi(0) \zeta^{4}$ & $\frac{\pi^{2}}{4 K^{4}}\left(\left(K^{2}-2\right)^{2} v_{\lambda}^{2}+4\left(K^{2}-2\right) v_{\lambda \mu}+4 v_{\mu}^{2}\right) \zeta^{2}$ \\
\hline $\bar{\Sigma}_{p s}(\zeta)$ & $\frac{16 \pi^{6} v_{\mu}^{2} \Phi(0)}{15 K K_{0}^{2}} \zeta^{4}$ & $\begin{array}{c}0 \text { for white noise and triangular } \\
\text { spectral for power-law and Gaussian } \\
\frac{2 v_{\mu}^{2}}{K K_{0}^{2}}\left(V_{2}^{-4}(K)-V_{4}^{-4}(K)\right) \text { for exponential }\end{array}$ \\
\hline $\bar{\Sigma}_{s s}(\zeta)$ & $\frac{4 \pi^{6} v_{\mu}^{2} \Phi(0)}{5} \zeta^{4}$ & $\frac{\pi^{2}}{4} v_{\mu}^{2} \zeta^{2}$ \\
\hline $\bar{\Sigma}_{p p}^{\prime}(\zeta)$ & $\begin{array}{c}0 \text { for white noise } \\
-\frac{16 \pi^{7}}{K^{4}}\left(\frac{1}{3}\left(K^{2}-2\right)^{2} v_{\lambda}^{2}+\frac{4}{5}\left(K^{2}-2\right) v_{\lambda \mu}+\frac{4}{7} v_{\mu}^{2}\right) \Phi^{\prime \prime}(0) \zeta^{6} \\
\text { for exponential and Gaussian } \\
\frac{8 \pi^{7}}{K^{4}}\left(\left(K^{2}-2\right)^{2} W_{1}^{1} v_{\lambda}^{2}+4\left(K^{2}-2\right) W_{3}^{1} v_{\lambda \mu}+4 W_{5}^{1} v_{\mu}^{2}\right) \Phi^{\prime}(0) \zeta^{5} \\
\text { for power-law and triangular }\end{array}$ & $\bar{\Sigma}_{p p}(\zeta \gg 1)$ \\
\hline $\bar{\Sigma}_{p s}^{\prime}(\zeta)$ & $\begin{array}{c}0 \text { for white noise } \\
-\frac{16 \pi^{6} v_{\mu}^{2} \Phi^{\prime \prime}(0)}{35 K K_{0}^{3}} \zeta^{6} \text { for exponential and Gaussian } \\
-\left(V_{5}^{1}(K)-V_{3}^{1}(K)\right) \frac{32 \pi^{7} \nu_{\mu}^{2} \Phi^{\prime}(0)}{K K_{0}^{2}} \zeta^{5} \text { for power-law and triangular }\end{array}$ & $\begin{array}{c}0 \text { for white noise and triangular } \\
\text { spectral for power-law and Gaussian } \\
\frac{2 v_{\mu}^{2}}{K K_{0}^{2}}\left(V_{3}^{-4}(K)-V_{5}^{-4}(K)\right) \text { for exponential }\end{array}$ \\
\hline $\bar{\Sigma}_{s s}^{\prime}(\zeta)$ & $\begin{array}{c}0 \text { for white noise } \\
-\frac{32 \pi^{8}}{105} v_{\mu}^{2} \Phi^{\prime \prime}(0) \zeta^{6} \text { for exponential and Gaussian } \\
4 \pi^{7}\left(W_{1}^{1}-3 W_{3}^{1}+4 W_{5}^{1}\right) v_{\mu}^{2} \Phi^{\prime}(0) \zeta^{5} \text { for power-law and triangular }\end{array}$ & $\bar{\Sigma}_{s s}(\zeta \gg 1)$ \\
\hline
\end{tabular}

\subsection{Influence of the correlation model on the forward scattering cross-sections}

We now turn to the forward scattering cross sections, that are defined as:

$$
\left\{\begin{array}{l}
\bar{\Sigma}_{p p}^{\prime}(\zeta)=\frac{4 \pi^{6}}{K^{4}}\left[\left(K^{2}-2\right)^{2} v_{\lambda}^{2} I_{1}(\zeta)+4\left(K^{2}-2\right) v_{\lambda \mu} I_{3}(\zeta)+4 v_{\mu}^{2} I_{5}(\zeta)\right] \zeta^{4} \\
\bar{\Sigma}_{p s}^{\prime}(\zeta)=\frac{16 \pi^{6}}{K K_{0}^{2}} v_{\mu}^{2}\left[J_{3}(\zeta)-J_{5}(\zeta)\right] \zeta^{4} \\
\bar{\Sigma}_{s s}^{\prime}(\zeta)=2 \pi^{6} v_{\mu}^{2}\left[I_{1}(\zeta)-3 I_{3}(\zeta)+4 I_{5}(\zeta)\right] \zeta^{4}
\end{array}\right.
$$

The evolutions of the forward scattering cross sections are plotted as a function of the normalized frequency $\zeta$ in Figure 5 and their asymptotic behavior described in Table 2. 


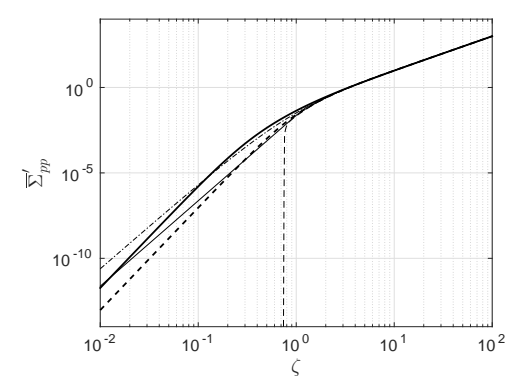

(a) $\bar{\Sigma}_{p p}^{\prime}$

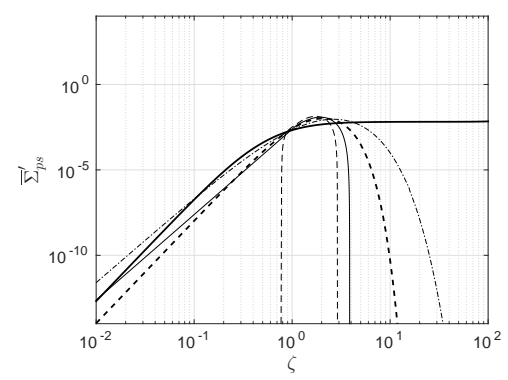

(b) $\bar{\Sigma}_{p s}^{\prime}$

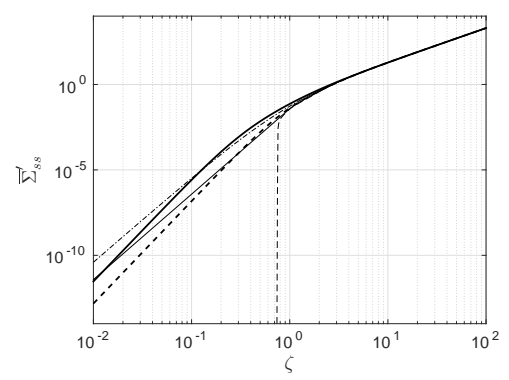

(c) $\bar{\Sigma}_{s s}^{\prime}$

Figure 5: $\bar{\Sigma}_{p p}^{\prime}, \bar{\Sigma}_{p s}^{\prime}$ and $\bar{\Sigma}_{s s}^{\prime}$ in terms of $\zeta$ for $K=\sqrt{3}$ and $v_{\lambda}=v_{\mu}=v_{\lambda \mu}=0.1$ : exponential (thick solid line), power-law (thin dashed-dotted line), Gaussian (thick dashed line), triangular (thin solid line) and low-pass white noise (thin dashed line).

It can be observed that the high frequency behavior of the forward scattering cross sections is very similar to that of the total scattering cross sections. Indeed, there is absolutely no difference between them for the P-to-P and S-to-S coefficients, and only the level of the plateau for the exponential correlation differs for the P-to-S cross section. This is compatible with the observations of the differential scattering cross-sections in Fig. 3 which showed a clear forward scattering tendency at high frequencies, for which the medium appears mostly homogeneous. In the low-frequency range, however, there is a larger influence of the correlation structure on the forward scattering cross section. The slope of the forward cross section depends (see Appendix A) on the first non-vanishing derivative of the power spectrum density. As all such derivatives vanish for the low-pass white noise model, there is a cut-off frequency below which all forward scattering cross sections vanish. This means that in the low frequency range for that correlation model, the incident waves are scattered equally in the forward and backward directions. For the exponential and Gaussian models, $\Phi^{\prime}(0)$ vanishes, while it does not for the power-law and triangular models. Hence the latter two models have more tendency to scatter waves in the forward direction in the low frequency range. Recalling that the derivatives of the power spectrum at the origin are related to the type of continuity (mean-square, almost-sure) of the realizations of a random process, it can be said that the value of the forward scattering cross-section is related to that degree of continuity. In any case, observing Figure 5, it can be concluded that there is little influence of the correlation model on the forward scattering cross sections, except in the high frequency range for the P-to-S cross section and in the low frequency range for the low-pass white noise model. In particular, there is little influence of the correlation model on the intermediate range $\zeta \approx 1$.

\section{Influence of the correlation function on the diffusion regime}

In this section, we study the influence of the correlation function on the scattering mean free path, transport mean free path and diffusion parameter.

\subsection{The diffusion regime}

For scalar waves in discrete random media, the scattering mean free path is the average distance between two scattering events. This notion is extended to continuous random media as the average distance before the wave direction is significantly altered. For elastic media, each propagation mode has its own scattering mean free path, and we normalize them with the correlation length:

$$
\ell_{\mathrm{P}}(\mathbf{k})=\ell_{c} \bar{\ell}_{p}\left(\frac{|\mathbf{k}| \ell_{c}}{\pi}\right), \quad \ell_{\mathrm{S}}(\mathbf{k})=\ell_{c} \bar{\ell}_{s}\left(\frac{|\mathbf{k}| \ell_{c}}{\pi}\right) .
$$

The influence of the correlation function on these normalized scattering mean free paths will be discussed in Section 4.2 .

Over longer propagation paths, and after many diffractions, the waves lose track of the source that created them. The average length for which this happens is denoted transport mean free path. In elastic media, there is one such 
path for each mode and we normalize them again with the correlation length:

$$
\ell_{\mathrm{P}}^{*}(\mathbf{k})=\ell_{c} \bar{\ell}_{p}^{*}\left(\frac{|\mathbf{k}| \ell_{c}}{\pi}\right), \quad \ell_{\mathrm{S}}^{*}(\mathbf{k})=\ell_{c} \bar{\ell}_{s}^{*}\left(\frac{|\mathbf{k}| \ell_{c}}{\pi}\right) .
$$

The influence of the correlation function on these normalized transport mean free paths will be discussed in Section 4.3.

Finally, it can be shown $[15,31]$ that after longer times, the energy density of the P-wave becomes isotropic $a^{p}(t, \mathbf{x}, \mathbf{k})=\phi(t, \mathbf{x},|\mathbf{k}|)$ and follows a diffusion equation

$$
\frac{\partial \phi}{\partial t}=\nabla_{\mathbf{x}} \cdot\left(D(|\mathbf{k}|) \nabla_{\mathbf{x}} \phi\right) .
$$

We normalize the diffusion parameter $D$ in the following manner

$$
D(|\mathbf{k}|)=\frac{\ell_{c} v_{p}}{1+2 K^{3}} \bar{D}\left(\frac{|\mathbf{k}| \ell_{c}}{\pi}\right)
$$

and we will discuss the influence of the correlation function on the normalized diffusion parameter in Section 4.4. Note that, in this diffusion regime, the $\mathrm{S}$ waves become depolarized and follow the same diffusion equation as the P-wave energy: $\left[a^{s}\left(t, \mathbf{x}, \mathbf{k}^{\prime}\right)\right]=\phi(t, \mathbf{x},|\mathbf{k}| / K) \mathbf{I}_{2}$.

\subsection{Influence on the scattering mean free path}

The normalized scattering mean free paths are defined as:

$$
\left\{\begin{array}{l}
\bar{\ell}_{p}(\zeta)=\left(\bar{\Sigma}_{p p}(\zeta)+4 \bar{\Sigma}_{p s}\left(\zeta \sqrt{K K_{0}}\right)\right)^{-1} \\
\bar{\ell}_{s}(\zeta)=\left(\bar{\Sigma}_{s s}(\zeta)+\frac{4}{K^{2}} \bar{\Sigma}_{p s}\left(\zeta K \sqrt{K K_{0}}\right)\right)^{-1}
\end{array}\right.
$$

Using the asymptotic results in Table 2, one can study the asymptotes of the mean free paths. They are summarized in Table 3. Plots of the scattering mean free paths for different values of $\zeta$ are also reported in Figure 6.

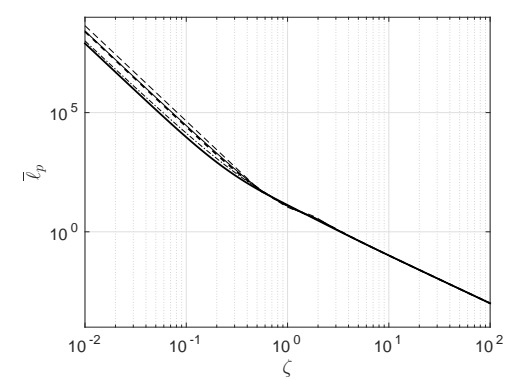

(a) $\bar{\ell}_{p}$

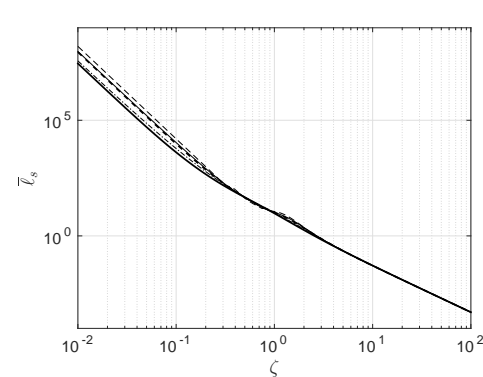

(b) $\bar{\ell}_{s}$

Figure 6: Scattering mean free paths $\bar{\ell}_{p}$ and $\bar{\ell}_{s}$ as functions of $\zeta$ for $K=\sqrt{3}$ and $v_{\lambda}=v_{\mu}=v_{\lambda \mu}=0.1$ : exponential (thick solid line), power-law (thin dashed-dotted line), Gaussian (thick dashed line), triangular (thin solid line) and low-pass white noise (thin dashed line).

Over the entire frequency range, the influence of the correlation structure on the two scattering mean free paths remains extremely limited. In the high frequency range, the cross term $\bar{\Sigma}_{p s}$ becomes negligible compared to both $\bar{\Sigma}_{p p}$ and $\bar{\Sigma}_{s s}$. As both these total scattering cross sections are independent of the correlation structure in the high frequency range, so are the scattering mean free paths. In the low-frequency range, the correlation structure only impacts the scattering mean free paths through the spectrum at the origin $\Phi(0)$. Note that a larger low-frequency content in the correlation kernel (larger $\Phi(0)$ ) induces a smaller scattering mean free path. Finally, we observe on Figure 6 that the influence of the correlation structure on the mean free paths in the medium frequency range is also negligible. 
Table 3: Asymptotic behavior of the scattering mean free path, transport mean free paths and diffusion parameter for different correlation models.

\begin{tabular}{|c|c|c|}
\hline Parameter & Low frequency $\zeta \ll 1$ & High frequency $\zeta \gg 1$ \\
\hline \hline $\bar{\ell}_{p}(\zeta)$ & $\frac{K^{4}}{2 \pi^{6}\left\{\left(K^{2}-2\right)^{2} v_{\lambda}^{2}+\frac{4}{3}\left(K^{2}-2\right) v_{\lambda \mu}+\frac{4}{15}\left(3+8 K^{5}\right) v_{\mu}^{2}\right\} \Phi(0)} \zeta^{-4}$ & $\bar{\Sigma}_{p p}^{-1}(\zeta \gg 1)$ \\
\hline $\bar{\ell}_{s}(\zeta)$ & $\frac{15}{2 \pi^{6}\left(3+16 K^{3}\right) v_{\mu}^{2} \Phi(0)} \zeta^{-4}$ & $\bar{\Sigma}_{s s}^{-1}(\zeta \gg 1)$ \\
\hline \hline $\bar{\ell}_{p}^{*}(\zeta)$ & $\bar{\ell}_{p}(\zeta \ll 1)$ & $\begin{array}{c}\text { logarithmic for exponential } \\
4 K^{4}\end{array}$ \\
\hline $\bar{\ell}_{s}^{*}(\zeta)$ & $\bar{\ell}_{s}(\zeta \ll 1)$ & $\frac{1}{\pi^{2}\left[\left(K^{2}-2\right)^{2} v_{\lambda}^{2}+4\left(K^{2}-2\right) v_{\lambda \mu}+4 v_{\mu}^{2}\right] \int_{0}^{\infty} p^{3} \Phi(p) d p}$ otherwise \\
\hline \hline $\bar{D}$ & $\frac{1}{16 \pi^{6}}\left[\frac{4}{\left(K^{2}-2\right)^{2} v_{\lambda}^{2}+\frac{4}{3}\left(K^{2}-2\right) v_{\lambda \mu}+\frac{4}{15}\left(3+8 K^{5}\right) v_{\mu}^{2}}+\frac{120 K^{2}}{\left(3+16 K^{3}\right) v_{\mu}^{2}}\right] \frac{\zeta^{-4}}{\Phi(0)}$ & $\frac{1}{\pi^{2} v_{\mu}^{2} \int_{0}^{\infty} p^{3} \Phi(p) d p}$ otherwise \\
\hline
\end{tabular}

\subsection{Influence on the transport mean free path}

The normalized transport mean free paths $\bar{\ell}_{p}^{*}$ and $\bar{\ell}_{s}^{*}$ are defined as:

$$
\left\{\begin{array}{l}
\bar{\ell}_{p}^{*}(\zeta)=\frac{\bar{\Sigma}_{s s}(\zeta)+\frac{1}{2 K^{2}} \bar{\Sigma}_{p s}\left(\zeta K \sqrt{K K_{0}}\right)-\bar{\Sigma}_{s s}^{\prime}(\zeta)+\bar{\Sigma}_{p s}^{\prime}\left(\zeta \sqrt{K K_{0}}\right)}{\left(\bar{\Sigma}_{p p}(\zeta)+\bar{\Sigma}_{p s}\left(\zeta \sqrt{K K_{0}}\right)-\bar{\Sigma}_{p p}^{\prime}(\zeta)\right)\left(\bar{\Sigma}_{s s}(\zeta)+\frac{1}{2 K^{2}} \bar{\Sigma}_{p s}\left(\zeta K \sqrt{K K_{0}}\right)-\bar{\Sigma}_{s s}^{\prime}(\zeta)\right)-\frac{1}{2 K^{2}} \bar{\Sigma}_{p s}^{\prime}\left(\zeta \sqrt{K K_{0}}\right) \bar{\Sigma}_{p s}^{\prime}\left(\zeta K \sqrt{K K_{0}}\right)} \\
\bar{\ell}_{p}^{*}(\zeta)=\frac{\bar{\Sigma}_{p p}(\zeta)+\bar{\Sigma}_{p s}\left(\zeta \sqrt{K K_{0}}\right)-\bar{\Sigma}_{p p}^{\prime}(\zeta)+\frac{1}{2 R^{2}} \bar{\Sigma}_{p s}^{\prime}\left(\zeta K \sqrt{K K_{0}}\right)}{\left(\bar{\Sigma}_{p p}(\zeta)+\bar{\Sigma}_{p s}\left(\zeta \sqrt{K K_{0}}\right)-\bar{\Sigma}_{p p}^{\prime}(\zeta)\right)\left(\bar{\Sigma}_{s s}(\zeta)+\frac{1}{2 K^{2}} \bar{\Sigma}_{p s}\left(\zeta K \sqrt{K K_{0}}\right)-\bar{\Sigma}_{s s}^{\prime}(\zeta)\right)-\frac{1}{2 K^{2}} \bar{\Sigma}_{p s}^{\prime}\left(\zeta \sqrt{K K_{0}}\right) \bar{\Sigma}_{p s}^{\prime}\left(\zeta K \sqrt{K K_{0}}\right)}
\end{array}\right.
$$

Although these formulas are more complex than the previous ones, it is interesting to note that in the particular case of non-preferential scattering, for which $\Sigma_{p p}^{\prime}=\Sigma_{p s}^{\prime}=\Sigma_{s s}^{\prime}=0$, we have $\bar{\ell}_{p}^{*}(\zeta)=\bar{\ell}_{p}(\zeta)$ and $\bar{\ell}_{s}^{*}(\zeta)=\bar{\ell}_{s}(\zeta)$. The asymptotes of the transport mean free paths are presented in Table 3. Plots of the transport mean paths for different values of $\zeta$ are also reported in Figure 6.

In the low frequency range, the forward scattering cross sections are negligible compared to the total cross sections, so that we retrieve the same asymptotic behavior for the transport mean free paths as for the scattering mean free paths. Hence, the correlation structure is only influential through the parameter $\Phi(0)$. In the high frequency range, the cross sections $\Sigma_{p s}$ and $\Sigma_{p s}^{\prime}$ become negligible compared to the other terms so that the transport mean free paths can be approximated as $\bar{\ell}_{p}^{*}(\zeta \gg 1) \approx\left(\bar{\Sigma}_{p p}(\zeta \gg 1)-\bar{\Sigma}_{p p}^{\prime}(\zeta \gg 1)\right)^{-1}$ and $\bar{\ell}_{s}^{*}(\zeta \gg 1) \approx\left(\bar{\Sigma}_{s s}(\zeta \gg 1)-\bar{\Sigma}_{s s}^{\prime}(\zeta \gg 1)\right)^{-1}$. However, the asymptotes considered until now are not sufficient so we have to consider higher order terms in the expansions of the integrals $I_{n}(\zeta \gg 1)$ (see Eq. (A.8)). The controlling parameter is the third moment of the PSDF $\int_{0}^{\infty} p^{3} \Phi(p) d p$, which highlights the high frequency content of the correlation kernel and is unbounded for the exponential correlation. Hence, the transport mean free paths converge to a constant for all correlation models, except for the exponential model, for which it converges to zero as $(\log \zeta)^{-1}$.

\subsection{Influence on the diffusion parameter}

The non-dimensional function $\bar{D}$ is defined as:

$$
\bar{D}(\zeta)=\bar{\ell}_{p}^{*}(\zeta)+2 K^{2} \bar{\ell}_{s}^{*}(\zeta)
$$

The behavior of the diffusion parameter as a function of $\zeta$ (see Figure 8) is essentially the same as that of the transport mean free paths so we will not comment any further on it. Asymptotic limits are given in Table 3.

\section{Conclusion}

In this paper, we discussed the influence of the correlation structure on the scattering cross sections, mean free paths and diffusion parameter, independently from that of the correlation length and variance. We considered five 


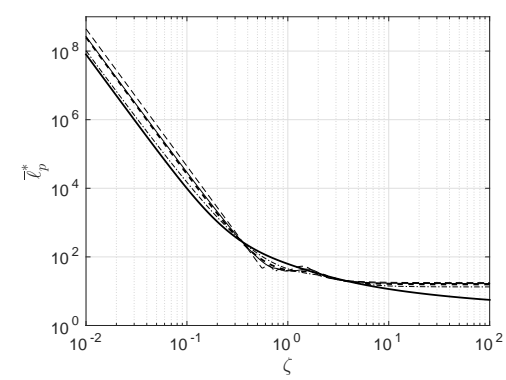

(a) $\bar{\ell}_{p}^{*}$

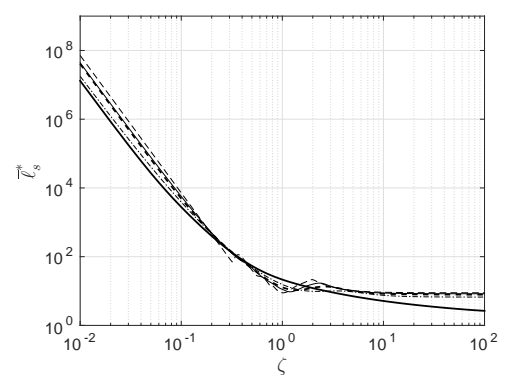

(b) $\bar{\ell}_{s}^{*}$

Figure 7: Transport mean free paths $\bar{l}_{p}^{*}$ and $\bar{l}_{s}^{*}$ as functions of $\zeta$ for $K=\sqrt{3}$ and $v_{\lambda}=v_{\mu}=v_{\lambda \mu}=0.1$ : exponential (thick solid line), power-law (thin dashed-dotted line), Gaussian (thick dashed line), triangular (thin solid line) and low-pass white noise (thin dashed line).

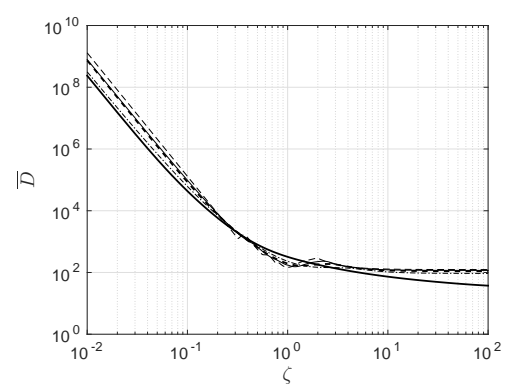

Figure 8: $\bar{D}$ in terms of $\zeta$ for $K=\sqrt{3}$ and $v_{\lambda}=v_{\mu}=v_{\lambda \mu}=0.1$ : exponential (thick solid line), power-law (thin dashed-dotted line), Gaussian (thick dashed line), triangular (thin solid line) and low-pass white noise (thin dashed line).

different correlation structures, with a wide range of behaviors. We identified that the controlling factors for the low-frequency behavior are the value of the PSDF $\Phi(0)$ and its first non-vanishing derivative at the origin $\left(\Phi^{\prime}(0)\right.$ for power-law and triangular correlations, $\Phi^{\prime \prime}(0)$ for Gaussian and exponential correlations, with a separate behavior for low-pass white noise correlation for which all derivatives at the origin vanish). In the high frequency range, the controlling factor is the third moment of the PSDF (which is unbounded for the exponential correlation). Overall, important differences between the correlation structures were only identified for some of the asymptotes for the total and forward scattering cross sections. For the rest of the cases, the influence of the correlation structure remains rather limited.

These conclusions may be useful in an identification process, by providing clues for the parameterization of the correlation structure. More specifically, this means that the quantity of information on the correlation kernel that can be inferred from energy measurements is rather limited. It will probably be possible to infer more than the correlation length and the variance only in the case where a rather large range of frequencies is available. In the low frequency range, it should be further stated that $\Phi(0)$ and $\sigma^{2}$ appear as a product, so it might not be possible to identify them independently one from the other. Contrarily, the derivatives of $\Phi(\zeta)$ at the origin and its third moment induce clear modifications of the tendencies so they might be more easily identified. As a conclusion for inverse problems based on energy quantities, the correlation model of a random media should, in most cases, be parameterized only with the correlation length and variance. Further, when the frequency range extends far enough towards the low frequencies, the value of $\Phi^{\prime}(\zeta)$ might be identifiable. When the frequency range extends far enough towards the high frequencies, the value of $\int_{0}^{\infty} p^{3} \Phi(p) d p$ might be identifiable. 


\section{Appendix A. Behavior of functions $I_{n}$ and $J_{n}$}

\section{Appendix A.1. Integral $W_{n}^{p}$}

We introduce, because it will be useful in understanding the behavior of the function $I_{n}$, the integral

$$
W_{n}^{p}=\int_{0}^{1}\left(1-2 \chi^{2}\right)^{n} \chi^{p+1} d \chi
$$

for $n \geq 0$ and $p \geq-1$. It can be shown that $W_{n}^{0}=\left(1+(-1)^{n}\right) / 4(n+1)$. Also, for $p \geq 1$, the following recurrence relation holds $(2 n+p+2) W_{n}^{p}=2 n W_{n-1}^{p}+(-1)^{n}$. Using the initial value $W_{0}^{p}=1 /(p+2)$, this means that the values $W_{n}^{p}$ can be computed for any pair $(n, p)$. In particular, we have $W_{n}^{2}=\left(1+(-1)^{n}(2 n+3)\right) / 8(n+1)(n+2)$.

Table A.4: Values of $W_{n}^{p}$ for the first values of $n$ and $p$.

\begin{tabular}{|c|c|c|c|c|c|}
\hline & $W_{n}^{0}$ & $W_{n}^{1}$ & $W_{n}^{2}$ & $W_{n}^{3}$ & $W_{n}^{4}$ \\
\hline$W_{0}^{p}$ & $1 / 2$ & $1 / 3$ & $1 / 4$ & $1 / 5$ & $1 / 6$ \\
\hline$W_{1}^{p}$ & 0 & $-1 / 15$ & $-1 / 12$ & $-3 / 35$ & $-1 / 12$ \\
\hline$W_{2}^{p}$ & $1 / 6$ & $11 / 105$ & $1 / 12$ & $23 / 315$ & $1 / 15$ \\
\hline$W_{3}^{p}$ & 0 & $-13 / 315$ & $-1 / 20$ & $-59 / 1155$ & $-1 / 20$ \\
\hline$W_{4}^{p}$ & $1 / 10$ & $211 / 3465$ & $1 / 20$ & $683 / 15015$ & $3 / 70$ \\
\hline$W_{5}^{p}$ & 0 & $-271 / 9009$ & $-1 / 28$ & $-1637 / 45045$ & $-1 / 28$ \\
\hline
\end{tabular}

\section{Appendix A.2. Integral $V_{n}^{p}$}

Similarly, we introduce for the understanding of $J_{n}$

$$
V_{n}^{p}(K)=K_{0}^{n+1} \int_{K_{1}}^{K_{2}}\left(1-2 \chi^{2}\right)^{n} \chi^{p+1} d \chi,
$$

for $n \geq 0$. We remind that $K_{0}=(K+1 / K) / 2, K_{1}=(K-1) / \sqrt{2\left(K^{2}+1\right)}$ and $K_{2}=(K+1) / \sqrt{2\left(K^{2}+1\right)}$. Note that $V_{n}^{p}(1)=W_{n}^{p}$. Using the binomial expansion, the values of $V_{n}^{p}$ can be calculated with

$$
V_{n}^{p}(K)=K_{0}^{n} \sum_{j=0}^{n}(-2)^{j}\left(\begin{array}{l}
n \\
j
\end{array}\right) V_{0}^{2 j+p}(K)
$$

and the values for $n=0$ which are $V_{0}^{-2}=K_{0} \log ((K+1) /(K-1))$ and $V_{0}^{p}=K_{0}\left(K_{2}^{p+2}-K_{1}^{p+2}\right) /(p+2)$ for $p \neq-2$. In particular, we have $V_{0}^{0}=1 / 2$ and $V_{0}^{-4}=2\left(\left(K^{2}+1\right) /\left(K^{2}-1\right)\right)^{2}$. With these relations, the values $V_{n}^{p}$ for any pair $(n, p)$ can be computed. They yield in particular $V_{n}^{0}=\left(1+(-1)^{n}\right) /(4(n+1))$ and $V_{2 n}^{2}=\left(1+(-1)^{n}\right) /(8(n+1))$. Observe that for these examples, $V_{n}^{p}$ is independent of $K$. This is however not true in general. Note also that, although it depends on the value of $K$, this family of parameters $V_{n}^{p}$ does not depend on the correlation kernel.

\section{Appendix A.3. Behavior of function $I_{n}$}

We consider the following integral function (see Figure A.9 for its behavior for different correlation functions and values of $n$ ):

$$
I_{n}(\zeta)=\int_{0}^{1}\left(1-2 \chi^{2}\right)^{n} \chi \Phi(2 \pi \zeta \chi) d \chi
$$

Using a Taylor expansion of the function $\Phi(2 \pi \zeta \chi)$ for low frequencies, ones gets $I_{n}(\zeta)=\sum_{p=0}^{\infty} \Phi^{(p)}(0)(2 \pi \zeta)^{p} W_{n}^{p} / p$ !, so that the limit behavior of $I_{n}(\zeta)$ is:

$$
I_{n}(\zeta \ll 1)=\frac{\Phi(0)}{2(n+1)}+\mathrm{O}(\zeta) \text { for even } n,
$$



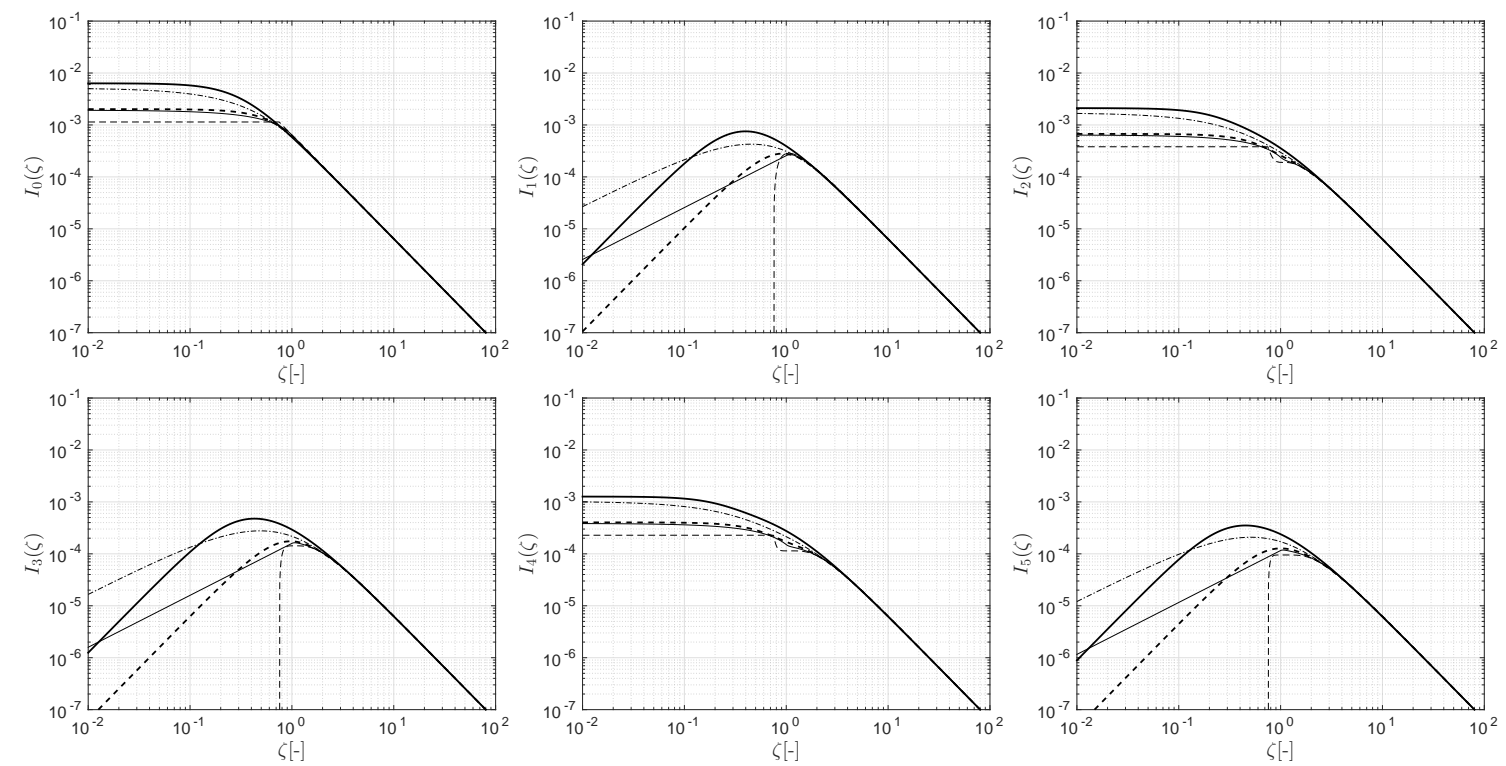

Figure A.9: Functions $I_{n}(\zeta)$ for different correlation kernels and different values of $n(K=\sqrt{3})$ : exponential (thick solid line), power-law (thin dashed-dotted line), Gaussian (thick dashed line), triangular (thin solid line) and low-pass white noise (thin dashed line).

and

$$
I_{n}(\zeta \ll 1)=2 \pi W_{n}^{1} \Phi^{\prime}(0) \zeta-\frac{\pi^{2} \Phi^{\prime \prime}(0)}{2(n+2)} \zeta^{2}+\mathrm{O}\left(\zeta^{3}\right) \text { for odd } n .
$$

Depending on the correlation model (see Table 1), $\Phi^{\prime}(0)$ sometimes cancels, so the expansion has to be continued up to second order. For the low-pass white noise model, for which the spectrum is flat and all its derivatives cancel, the expansion is not correct. However, in that case, we can compute explicitly $I_{n}^{\text {noise }}(\zeta)$ :

$$
I_{n}^{\text {noise }}(\zeta \leq 3 / 4)=\frac{1}{18 \pi^{4}(n+1)}\left(1+(-1)^{n}\right), \quad \text { and } \quad I_{n}^{\text {noise }}(\zeta \geq 3 / 4)=\frac{1}{18 \pi^{4}(n+1)}\left(1-\left(1-\frac{9 \pi^{2}}{8 \zeta^{2}}\right)^{n+1}\right) .
$$

The high-frequency behavior of the function $I_{n}(\zeta)$ can be investigated through the change of variable $2 \pi \zeta \chi=p$ :

$$
I_{n}(\zeta \gg 1)=\frac{1}{(2 \pi \zeta)^{2}} \int_{0}^{2 \pi \zeta}\left(1-\frac{2 p^{2}}{(2 \pi \zeta)^{2}}\right)^{n} p \Phi(p) d p=\frac{1}{16 \pi^{4}} \zeta^{-2}-\frac{n}{16 \pi^{4}} \zeta^{-4} \int_{\mathbb{R}^{+}} p^{3} \Phi(p) d p+\mathrm{O}\left(\zeta^{-6}\right)
$$

This limit is hence independent at first order from both the correlation kernel and $n$. When looking for the difference $I_{n}(\zeta)-I_{n+1}(\zeta)$ however, one must resort to second order analysis, since only the second term depends on $n$. At that order, we have for the exponential correlation structure $8 \pi^{2} \int_{0}^{2 \pi \zeta} p^{3} \Phi(p) d p \approx \log \zeta$ for $\zeta \gg 1$. The results obtained from the asymptotic analysis are summarized in Table A.5.

\section{Appendix A.4. Behavior of function $J_{n}$}

We finally consider the following integral function (see Figure A.10 for its behavior for different correlation functions and values of $n$ ):

$$
J_{n}(\zeta ; K)=K_{0}^{n+1} \int_{K_{1}}^{K_{2}}\left(1-2 \chi^{2}\right)^{n} \chi \Phi(2 \pi \zeta \chi) d \chi
$$

Note that this function is related to the previous one through $J_{n}(\zeta ; 1)=I_{n}(\zeta)$.

As before we use the Taylor expansion $J_{n}(\zeta ; K)=\sum_{p=0}^{\infty} \Phi^{(p)}(0)(2 \pi \zeta)^{p} V_{n}^{p} / p$ ! and hence derive the low-frequency behavior:

$$
J_{n}(\zeta \ll 1 ; K)=\frac{\Phi(0)}{2(n+1)}+\mathrm{O}(\zeta) \text { for even } n,
$$


Table A.5: Asymptotic values of $I_{n}(\zeta), J_{n}(\zeta ; K)$ and several useful combinations

\begin{tabular}{|c|c|c|}
\hline Integral & Low frequency $\zeta \ll 1$ & High frequency $\zeta \gg 1$ \\
\hline$I_{n}(\zeta)$ & For odd $n: \begin{cases} & \text { For even } n: \frac{\Phi(0)}{2(n+1)} \\
-\frac{\pi^{2} \Phi^{\prime \prime}(0)}{2(n+2)} \zeta^{2} & \text { for white noise } \\
2 \pi W_{n}^{1} \Phi^{\prime}(0) \zeta & \text { for powenential and Gaussian }\end{cases}$ & $\frac{1}{16 \pi^{4}} \zeta^{-2}$ \\
\hline$J_{n}(\zeta ; K)$ & For odd $n: \begin{cases}0 & \text { For even } n: \frac{\Phi(0)}{2(n+1)} \\
-\frac{\pi^{2} \Phi^{\prime \prime}(0)}{2(n+2) K_{0}} \zeta^{2} & \text { for white exponential and Gaussian } \\
2 \pi V_{n}^{1} \Phi^{\prime}(0) \zeta & \text { for power-law and triangular }\end{cases}$ & $\begin{array}{l}0 \text { for white noise and triangular } \\
\text { spectral for power-law and Gaussian } \\
\frac{1}{8 \pi^{6}} V_{n}^{-4} \zeta^{-4} \text { for exponential }\end{array}$ \\
\hline$I_{n}-I_{n+1}($ even $n)$ & $I_{n}(\zeta \ll 1)$ & $\begin{array}{l}\frac{1}{128 \pi^{6}} \zeta^{-4} \log \zeta \text { for exponential } \\
\frac{1}{16-4} \zeta^{-4} \int_{1+ \pm} p^{3} \Phi(p) d p \text { otherwise }\end{array}$ \\
\hline$I_{0}(\zeta)-3 I_{2}(\zeta)+4 I_{4}(\zeta)$ & $\frac{2}{5} \Phi(0)$ & $\frac{1}{8 \pi^{4}} \zeta^{-2}$ \\
\hline$J_{2}(\zeta ; K)-J_{4}(\zeta ; K)$ & $\frac{1}{15} \Phi(0)$ & $\begin{array}{c}0 \text { for white noise and triangular } \\
\text { spectral for power-law and Gaussian } \\
\frac{1}{8 \pi^{6}}\left(V_{2}^{-4}(K)-V_{4}^{-4}(K)\right) \zeta^{-4} \text { for exponential }\end{array}$ \\
\hline$I_{1}(\zeta)-3 I_{3}(\zeta)+4 I_{5}(\zeta)$ & $\begin{array}{c}0 \text { for white noise } \\
-\frac{16 \pi^{2}}{105} \Phi^{\prime \prime}(0) \zeta^{2} \text { for exponential and Gaussian } \\
2 \pi\left(W_{1}^{1}-3 W_{3}^{1}+4 W_{5}^{1}\right) \Phi^{\prime}(0) \zeta \text { for power-law and triangular }\end{array}$ & $\frac{1}{8 \pi^{4}} \zeta^{-2}$ \\
\hline$J_{3}(\zeta ; K)-J_{5}(\zeta ; K)$ & $\begin{array}{c}0 \text { for white noise } \\
-\frac{\pi^{2} \Phi^{\prime \prime}(0)}{35 K_{0}} \zeta^{2} \text { for exponential and Gaussian } \\
2 \pi\left(V_{3}^{1}(K)-V_{5}^{1}(K)\right) \Phi^{\prime}(0) \zeta \text { for power-law and triangular }\end{array}$ & $\begin{array}{c}0 \text { for white noise and triangular } \\
\text { spectral for power-law and Gaussian } \\
\frac{1}{8 \pi^{6}}\left(V_{3}^{-4}(K)-V_{5}^{-4}(K)\right) \zeta^{-4} \text { for exponential }\end{array}$ \\
\hline
\end{tabular}
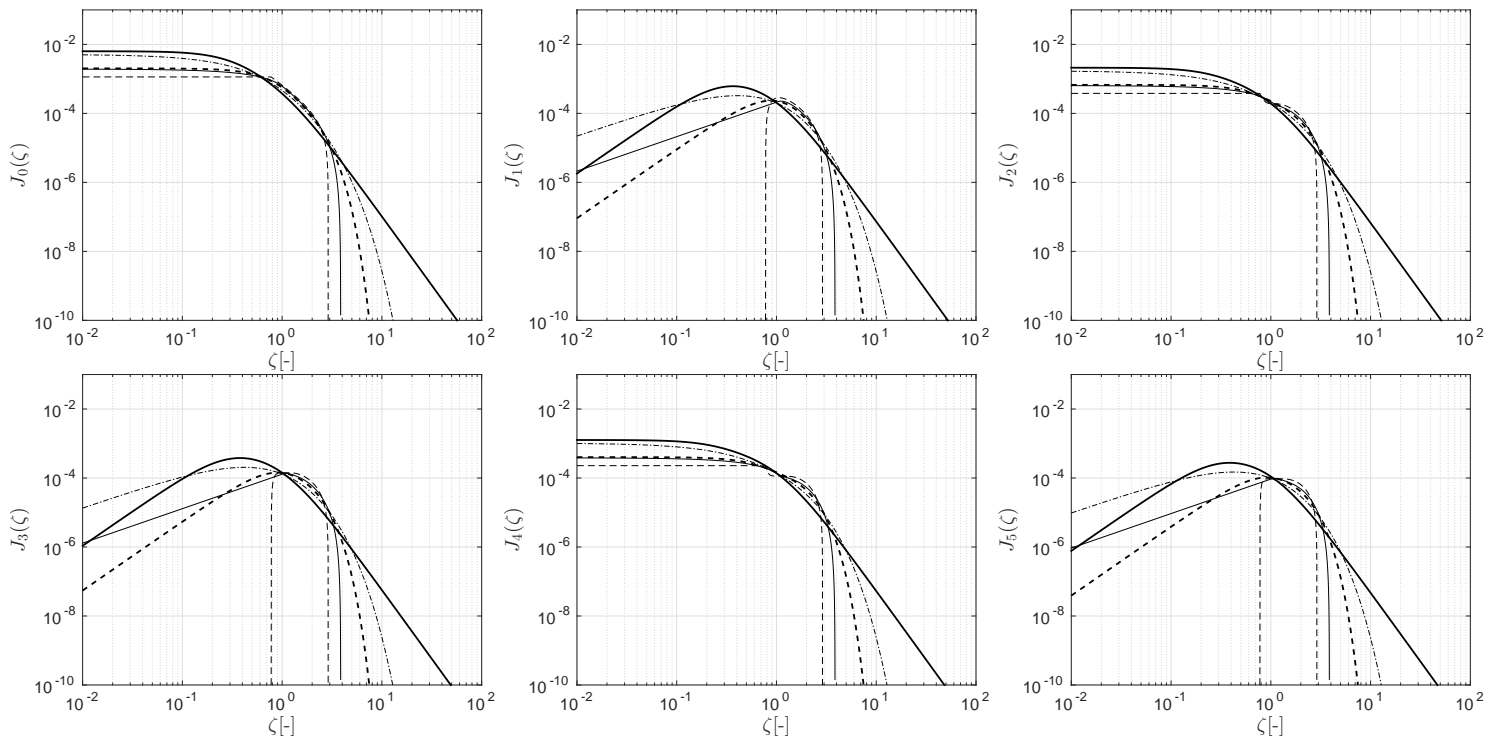

Figure A.10: Functions $J_{n}(\zeta)$ for different correlation kernels and different values of $n(K=\sqrt{3})$ : exponential (thick solid line), power-law (thin dashed-dotted line), Gaussian (thick dashed line), triangular (thin solid line) and low-pass white noise (thin dashed line). 
and

$$
J_{n}(\zeta \ll 1 ; K)=2 \pi V_{n}^{1} \Phi^{\prime}(0) \zeta-\frac{\pi^{2} \Phi^{\prime \prime}(0)}{2(n+2) K_{0}} \zeta^{2}+\mathrm{O}\left(\zeta^{3}\right) \text { for odd } n .
$$

These expansions do not apply to the white noise case, but this case can be computed explicitly:

$$
J_{n}^{\text {noise }}\left(\zeta \leq \frac{3}{4 K_{2}}\right)=\frac{1}{18 \pi^{4}(n+1)}\left(1+(-1)^{n}\right), \quad J_{n}^{\text {noise }}\left(\frac{3}{4 K_{2}} \leq \zeta \leq \frac{3}{4 K_{1}}\right)=\frac{1}{18 \pi^{4}(n+1)}\left(1-K_{0}^{n+1}\left(1-\frac{9 \pi^{2}}{8 \zeta^{2}}\right)^{n+1}\right)
$$

and $J_{n}^{\text {noise }}\left(\zeta \geq 3 / 4 K_{1}\right)=0$ which also provides the high-frequency behavior of $J$ for a low-pass white noise correlation model. Note that, for odd $n, J_{n}^{\text {noise }}$ vanishes both in the low and high frequency ranges (outside the interval $\left.\left[3 / 4 K_{2}, 3 / 4 K_{1}\right]\right)$.

Likewise, for the case of a random medium with triangular correlation, one can compute explicitly the values of the integral for the low and high frequency ranges:

$$
J_{n}^{\text {tri }}\left(\zeta \leq \frac{1}{K_{2}}\right)=\frac{3}{8 \pi^{4}}\left(V_{n}^{0}-\zeta V_{n}^{1}\right)
$$

and $J_{n}^{\text {tri }}\left(\zeta \geq 1 / K_{1}\right)=0$. Note that $V_{n}^{0}=0$ for odd $n$ so that the asymptotic is then given by the linear term. The slope is positive because $V_{n}^{1}<0$ for odd $n$.

For the exponential model, we have in the high frequency range $\Phi(2 \pi \zeta \chi)=\zeta^{-4} \chi^{-4} /\left(8 \pi^{6}\right)+o\left(\zeta^{-4}\right)$ so that

$$
J_{n}^{\exp }(\zeta \gg 1 ; K)=\frac{1}{8 \pi^{6}} V_{n}^{-4} \zeta^{-4}+\mathrm{o}\left(\zeta^{-4}\right)
$$

Finally, for both the power-law and Gaussian models, the $J_{n}(\zeta \gg 1 ; K)$ goes to zero exponentially fast.

\section{References}

[1] K. Aki, Analysis of the seismic coda of local earthquakes as scattered waves, Journal of Geophysical Research 74 (1969) 615-631.

[2] M. Herraiz, A. F. Espinosa, Coda waves: a review, Pure and Applied Geophysics 125 (1987) 499-577.

[3] H. Sato, M. Fehler, T. Maeda, Seismic Wave Propagation and Scattering in the Heterogeneous Earth: Second Edition, SpringerLink : Bücher, Springer Berlin Heidelberg, 2012.

[4] R. L. Weaver, On diffuse waves in solid media, Journal of the Acoustical Society of America 71 (1982) 1608-1609.

[5] K. Aki, B. Chouet, Origin of coda waves: Source, attenuation, and scattering effects, Journal of Geophysical Research 80 (1975) $3322-3342$.

[6] A. Pitarka, G. Ichinose, Simulating forward and backward scattering in viscoelastic 3D media with random velocity variations and basin structure, Technical Report 06HQGR0042, USGS, 2010.

[7] S. Hartzell, S. Harmsen, A. Frankel, Effects of 3D random correlated velocity perturbations on predicted ground motions, Bulletin of the Seismological Society of America 100 (2010) 1415-1426.

[8] Q.-A. Ta, D. Clouteau, R. Cottereau, Modeling of random anisotropic elastic media and impact on wave propagation, European Journal of Computational Mechanics 19 (2010) 241-253.

[9] W. Imperatori, P. M. Mai, Broad-band near-field ground motion simulations in 3-dimensional scattering media, Geophysical Journal International 192 (2013) 725-744.

[10] S. Takemura, T. Furumura, T. Maeda, Scattering of high-frequency seismic waves caused by irregular surface topography and small-scale velocity inhomogeneity, Geophysical Journal International 201 (2015) 459-474.

[11] S. Chandrasekhar, Radiative transfer, Dover Publications, 1960.

[12] A. Ishimaru, Wave Propagation and Scattering in Random Media, Academic Press, 1978.

[13] P. Sheng, Introduction to Wave Scattering, Localization, and Mesoscopic Phenomena, Academic Press, San Diego, 1995.

[14] J.-P. Fouque, J. Garnier, G. Papanicolaou, Wave Propagation and Time Reversal in Randomly Layered Media, Stochastic Modelling and Applied Probability, Springer, Dordrecht, 2007.

[15] R. L. Weaver, Diffusivity of ultrasound in polycrystals, Journal of the Mechanics and Physics of Solids 38 (1990) 55-86.

[16] J. A. Turner, R. L. Weaver, Radiative transfer and multiple scattering of diffuse ultrasound in polycrystalline media, Journal of the Acoustical Society of America 96 (1994) 3675-3683.

[17] T. J. Schultz, Diffusion in reverberation rooms, Journal of Sound and Vibration 16 (1971) 17-28.

[18] K. Bodlund, A study of diffusion in reverberation chambers provided with special devices, Journal of Sound and Vibration 50 (1977) 253-283.

[19] N. L. Wolff, R. L. Weaver, Towards a diffusion model of acoustic energy flow in large undamped structures, Journal of Sound and Vibration 288 (2005) 729-749.

[20] E. Savin, High-frequency dynamics of heterogeneous slender structures, Journal of Sound and Vibration 332 (2013) $2461-2487$.

[21] P. Anugonda, J. S. Wiehn, J. A. Turner, Diffusion of ultrasound in concrete, Ultrasonics 39 (2001) 429-435. 
[22] K. Busch, C. M. Soukoulis, E. N. Economou, Transport and scattering mean free paths of classical waves, Physical Review B 50 (1994) 93-98.

[23] R. L. Weaver, W. Sachse, Diffusion of ultrasound in glass bead slurry, Journal of the Acoustical Society of America 97 (1995) 2094.

[24] V. Tournat, V. E. Gusev, B. Castagnède, Influence of ballistics to diffusion transition in primary wave propagation on parametric antenna operation in granular media, Physical Review E 66 (2002) 1-10.

[25] G. E. Backus, Long-wave elastic anisotropyc produced by horizontal layering, Journal of Geophysical Research 67 (1962) $4427-4440$.

[26] Y. Capdeville, L. Guillot, J. J. Marigo, 1-d non-periodic homogenization for the seismic wave equation, Geophysical Journal International 2 (2010) 897-910.

[27] C. Pierre, Weak and strong vibration localization in disordered structures: a statistical investigation, Journal of Sound and Vibration 139 (1990) 111-132.

[28] R. L. Weaver, O. I. Lobkis, Anderson localization in coupled reverberation rooms, Journal of Sound and Vibration 231 (2000) $1111-1134$.

[29] Y. Zeng, Theory of scattered P-wave and S-wave energy in a random isotropic scattering medium, Bulletin of the Seismological Society of America 83 (1993) 1264-1276.

[30] H. Sato, Multiple isotropic scattering model including P-S conversions for the seismogram envelope formation, Geophysical Journal International 117 (1994) 487-494.

[31] L. Ryzhik, G. Papanicolaou, J. B. Keller, Transport equations for elastic and other waves in random media, Wave Motion 24 (1996) 327-370.

[32] J. A. Turner, Scattering and diffusion of seismic waves, Bulletin of the Seismological Society of America 88 (1998) $276-283$.

[33] D. Liu, J. A. Turner, Influence of spatial correlation function on attenuation of ultrasonic waves in two-phase materials, Journal of the Acoustical Society of America 123 (2008) 2570-2576.

[34] U. Frisch, Wave propagation in random media, in: A. T. Bharucha-Reid (Ed.), Probabilistic Methods in Applied Mathematics, volume 1, 1968, pp. 75-198.

[35] H. Sato, H. Nakahara, M. Ohtake, Synthesis of scattered energy density for nonspherical radiation from a point shear-dislocation source based on the radiative transfer theory, Physics of the Earth and Planetary Interiors 104 (1997) 1-13.

[36] F. J. Herrmann, Multiscale analysis of well and seismic data, in: S. Hassanzadeh (Ed.), Proceedings of SPIE Conference on Mathematical Methods in Geophysical Imaging V, volume 3453, 1998, pp. 180-203. doi:10.1117/12.323290.

[37] N. P. Trégourès, B. A. van Tiggelen, Generalized diffusion equation for multiple scattered elastic waves, Waves in Random Media 12 (2002) 21-38.

[38] N. L. Bihan, L. Margerin, Nonparametric estimation of the heterogeneity of a random medium using compound poisson process modeling of wave multiple scattering, Physical Review E 80 (2009) 1-9.

[39] I. Baydoun, E. Savin, R. Cottereau, D. Clouteau, J. Guilleminot, Kinetic modeling of multiple scattering of elastic waves in heterogeneous anisotropic media, Wave Motion 51 (2014) 1325-1348.

[40] J. A. Turner, P. Anugonda, Scattering of elastic waves in heterogeneous media with local isotropy, Journal of the Acoustical Society of America 109 (2011) 1787-1795.

[41] H. Sato, Attenuation and envelope formation of three-component seismograms of small local earthquakes in randomly inhomogeneous lithosphere, Journal of Geophysical Research 89 (1984) 1221-1241.

[42] L. Klimeš, Correlation functions of random media, Pure and Applied Geophysics 159 (2002) 1811-1831. 


\section{List of Figures}

1 Classical correlation functions (left figure) and corresponding power spectral densities (right figure), as described in Table 1: exponential (thick solid line), power-law (thin dashed-dotted line), Gaussian (thick dashed line), triangular (thin solid line) and low-pass white noise (thin dashed line). . . . . . .

2 One realization of a centered unit Gaussian random field with different correlation models: (a) exponential, (b) power-law, (c) Gaussian, (d) triangular, and (e) low-pass white noise. The images are

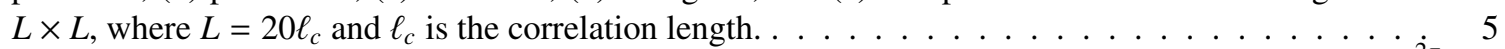

3 Influence of the correlation model on the scattering probability density functions $\hat{\sigma}_{i j}(\zeta, \theta)=\bar{\sigma}_{i j}(\zeta, \theta) / \int_{0}^{2 \pi} \bar{\sigma}_{i j}(\zeta, \theta) d \theta$ for $K=\sqrt{3}$ and $v_{\lambda}=v_{\mu}=v_{\lambda \mu}=0.1$ : exponential (thick solid line), power-law (thin dashed-dotted line), Gaussian (thick dashed line), triangular (thin solid line) and low-pass white noise (thin dashed

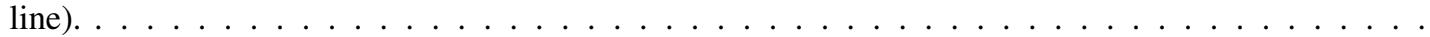

$4 \bar{\Sigma}_{p p}, \bar{\Sigma}_{p s}$ and $\bar{\Sigma}_{s s}$ in terms of $\zeta$ for $K=\sqrt{3}$ and $v_{\lambda}=v_{\mu}=v_{\lambda \mu}=0.1$ : exponential (thick solid line), power-law (thin dashed-dotted line), Gaussian (thick dashed line), triangular (thin solid line) and low-pass white noise (thin dashed line) . . . . . . . . . . . . . . . . . . . .

$5 \quad \bar{\Sigma}_{p p}^{\prime}, \bar{\Sigma}_{p s}^{\prime}$ and $\bar{\Sigma}_{s s}^{\prime}$ in terms of $\zeta$ for $K=\sqrt{3}$ and $v_{\lambda}=v_{\mu}=v_{\lambda \mu}=0.1$ : exponential (thick solid line), power-law (thin dashed-dotted line), Gaussian (thick dashed line), triangular (thin solid line) and low-pass white noise (thin dashed line) . . . . . . . . . . . . . . . . . .

6 Scattering mean free paths $\bar{\ell}_{p}$ and $\bar{\ell}_{s}$ as functions of $\zeta$ for $K=\sqrt{3}$ and $\nu_{\lambda}=v_{\mu}=v_{\lambda \mu}=0.1$ : exponential (thick solid line), power-law (thin dashed-dotted line), Gaussian (thick dashed line), triangular (thin solid line) and low-pass white noise (thin dashed line).

Transport mean free paths $\bar{l}_{p}^{*}$ and $\bar{l}_{s}^{*}$ as functions of $\zeta$ for $K=\sqrt{3}$ and $v_{\lambda}=v_{\mu}=v_{\lambda \mu}=0.1$ : exponential (thick solid line), power-law (thin dashed-dotted line), Gaussian (thick dashed line), triangular (thin solid line) and low-pass white noise (thin dashed line) . . . . . . . . . . . . . . . . .

$8 \bar{D}$ in terms of $\zeta$ for $K=\sqrt{3}$ and $v_{\lambda}=v_{\mu}=v_{\lambda \mu}=0.1$ : exponential (thick solid line), power-law (thin dashed-dotted line), Gaussian (thick dashed line), triangular (thin solid line) and low-pass white noise (thin dashed line $). \ldots \ldots \ldots \ldots \ldots \ldots \ldots \ldots$

A.9 Functions $I_{n}(\zeta)$ for different correlation kernels and different values of $n(K=\sqrt{3})$ : exponential (thick solid line), power-law (thin dashed-dotted line), Gaussian (thick dashed line), triangular (thin solid line) and low-pass white noise (thin dashed line). . . . . . . . . . . . . . . . . . .

A.10 Functions $J_{n}(\zeta)$ for different correlation kernels and different values of $n(K=\sqrt{3})$ : exponential (thick solid line), power-law (thin dashed-dotted line), Gaussian (thick dashed line), triangular (thin solid line) and low-pass white noise (thin dashed line) . . . . . . . . . . . . . . . . 